\title{
Present Status of Orchid Species Diversity Resources of Joypur Reserve Forest of Dibrugarh District (Assam) of North East India
}

\author{
Khyanjeet Gogoi $^{1, *}$, R. L. Borah ${ }^{2}$, Raju Das ${ }^{3}$, Rajendra Yonzone ${ }^{4}$ \\ ${ }^{1}$ Daisa Bordoloi Nagar, Talap, Tinsukia , 786156, Assam, India \\ ${ }^{2}$ Dept. of Botany, DHSK College, Dibrugarh, 786001, Assam, India \\ ${ }^{3}$ Nature's Foster, P. Box 41, Shastri Road, P.O. Bongaigaon, 783380, Assam, India \\ ${ }^{4}$ Dept. of Botany, St. Joseph's College, North Point, district Darjeeling, W. B. , 734104, India
}

\begin{abstract}
Present paper deals with the Orchid species diversity of Joypur reserve forest of Dibrugarh district of Assam of North East India. A total of 107 Orchid species with 47 genera have been recorded from the region. Of them, 82 are epiphytic, 23 are terrestrial and 2 species like Didymoplexis pallens and Epipogium roseum are saprophytic. This attempt is the first step to correct taxonomic identification to workout currently accepted botanical names, voucher specimen, habitat, phenology and locality of Orchid species occurring in the study regions.
\end{abstract}

Keywords Orchid Species Diversity, Joypur Reserve Forest, Dibrugarh District, Assam

\section{Introduction}

Orchids belong to the family Orchidaceae is considered to be the most highly evolved in the floral specialization and diversified form among the monocotyledons. In India, Orchids from $9 \%$ of our flora and are the largest and highly advanced botanical family of higher plants. It is estimated that at about 25,000-35,000 species with 800-1,000 genera are distributed throughout the world. About 1331 species with 140 genera of Orchid species are found in India with temperate Himalayas as their natural home[23].

\section{Study area}

Joypur is a Reserve forest endowed with rich Biodiversity especially in orchids besides other plant species, which is also a part of one of the biodiversity hot spot[11]. It is situated between $95^{\circ} 22^{\prime}$ E. to $95^{\circ} 30^{\prime} \mathrm{E}$. and $27^{\circ} 00^{\prime} \mathrm{N}$. to $27^{\circ} 16^{\prime} \mathrm{N}$. covering a geographical area of $10,876.68$ hectares (Figure 1). It forms a part of the world heritage of tropical/sub-tropical wet evergreen forest, multistoried in structure and rich in biodiversity, more popularly known as 'Rain forest'. The forest is rich in biodiversity and one of the great reservoirs of Orchid germplasm due to its high rainfall, relative humidity etc. (Gogoi et al, 2009, 2010).

Present investigation reports the Orchid species diversity resources of Joypur Reserve Forest of Dibrugarh district of

* Corresponding author:

khyanjeetgogoi@gmail.com (Khyanjeet Gogoi)

Published online at http://journal.sapub.org/ijmb

Copyright (C) 2012 Scientific \& Academic Publishing. All Rights Reserved
Assam with voucher specimen, habitat, date of collection, phenology, local distribution with Joypur Reserve Forest and general distribution.

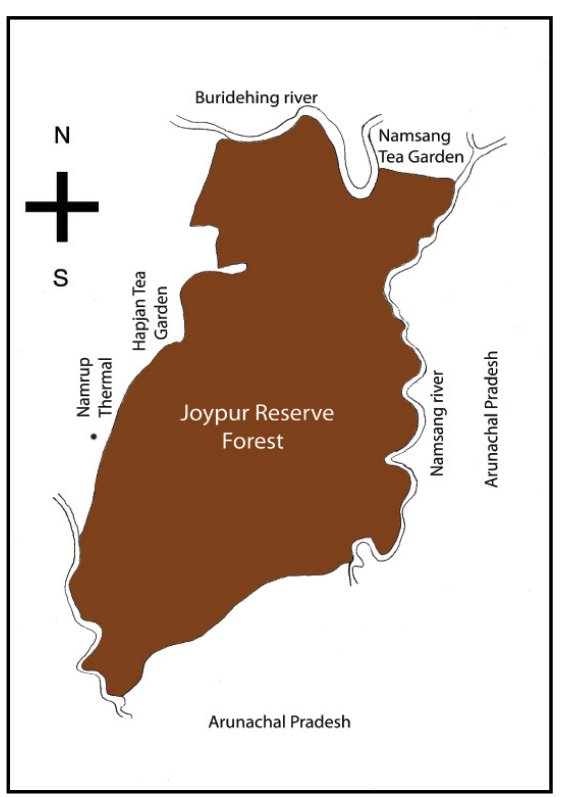

Figure 1. Location of study area (Joypur Reserve Forest of Dibrugarh district, Assam)

\section{Materials and Methods}

The intensive field survey works were carried out during 2009-2011 covering all the seasons of the year in all parts of Joypur reserve forest of Dibrugarh district of Assam. 
Collected specimens were made into standard mounted herbarium sheets following the procedure of Jain and Rao[15]. The authors have done photographs and sketches of all the available Orchid species of the region. The relevant data from the field notebooks were then transferred to the labels of the herbarium sheets and computer. Normally, 2-3 specimens of each species in flowering or fruiting stage were collected and life form photographs were prepared. The specimens were identified, authenticate and described with the help of The specimens were identified, described and nomenclature checked with the help of The genera and species of Orchidaceous plants[21]; Folia Orchidacea[20]; The orchids of the Sikkim Himalayas[16]; A Guide to the Orchid of Sikkim[4]; The Orchid Flora of North-West Himalaya[8]; Notes on Cirrhopetalum[30]; Indian Orchids Guide to Identificationand Culture[27]; Indian Orchids Guide to Identificationand Culture[28]; Orchids ofMussoorie[29]; Orchids of Arunachal Pradesh[13]; Flora of British India[14]; Orchids of Kumaun Himalaya[24]; A Catalogue of Indian Orchids[17]; Orchids of Nagaland[7]; Orchid Flora of Arunachal Pradesh[5]; 100 Sikkim Himalayan Orchids[26] etc. and by matching at the Herbarium of the Department of Botany, Guwahati University. Finally all the Voucher specimens are deposited in the Herbarium, Department of Botany, Guwahati University.

\section{Enumeration}

\section{Acampe Lindley}

About 10 species in India, China, S. E. Asia and tropical Africa; 6 species in India, 3 species in Assam, 2 in Joypur Reserve Forest.

Acampe papillosa (Lindl.) Lindl., Fol. Orchid, 2, 1853; Chowdhery, Orch. Fl. Arunachal Prad. 41. 1998.

Plant $8-23 \mathrm{~cm}$ tall with many distichous leaves. Leaf 7-14.5×1.4-2.3cm, oblong. Inflorescence several, opposite to leaves or axillary, $1-4 \mathrm{~cm}$ long. Flowers $1 \mathrm{~cm}$ across, yellow, slightly fragrant; sepals and petals yellow with reddish brown transverse stripes; lip white, slightly spotted with purple-red.

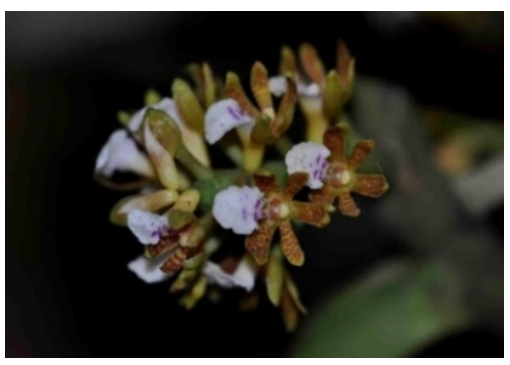

Figure 2. Acampe papillosa

Habitat: Epiphyte on tree trunk. Voucher specimen: Gogoi et al. 0226. Flowering: November - December. Local distribution: Joypur R. F, Assam. General distribution: India, China, Nepal, Burma, Thailand, Vietnam, Bangladesh, Bhutan, Laos, Myanmar. (Figure 2)
Acampe rigida (Buch.-Ham. ex J.E. Smith) P.F. Hunt in Kew Bull. 24: 98. 1970; Chowdhery, Orch. Fl. Arunachal Prad. 41, 1998; Mishra, Orch. India 280. 2007.

Plants $61-86 \mathrm{~cm}$ long. Stem $0.8-1 \mathrm{~cm}$ thick, erect, unbranc hed or branched. Leaves $14-26 \times 3.5-4.1 \mathrm{~cm}$, distichous, fleshy-coriaceous, conduplicate, curved, linear-oblong, apex unequally 2-lobed. Inflorescence axillary, subcapitate, paniculate, densely 7-11 flowered; peduncle $5-7 \mathrm{~cm}$ long. Flowers yellow with purplish brown transverse stripes; lip white, with purplish brown longitudinal stripes above.

Habitat: Epiphyte on tree trunks or large branches. Voucher specimen: Gogoi et al. 0096. Flowering: June - July. Local distribution: Joypur R. F, Assam. General distribution: Southern and east Africa, Sri Lanka, India, Burma, China, Taiwan, Thailand, Cambodia, Vietnam, Peninsular Malaysi a.

\section{Acanthephippium Blume}

About 15 species in tropical Asia, Malaysia and Fiji; 3 species in India, 2 in Assam and 1 species in Joypur Reserve Forest.

Acanthephippium sylhetense Lindl., Gen. Sp. Orchid. Pl. 177. 1833; Hook. f., Fl. Brit. India 5: 815. 1890; Chowdhery, Orch. Fl. Arunachal Prad. 43. 1998.

Plants to $50 \mathrm{~cm}$ tall. Pseudobulb ovoidcylindric, $7-15 \times$ 1.5-4cm, 2-4-noded, 2-4 leaved. Leaves elliptic to oblongelliptic, $30-40 \times 8-11 \mathrm{~cm}$, apex acuminate. Inflorescence $15-20 \mathrm{~cm}$, fleshy, with several broadly ovate-lanceolate sheaths at base; rachis densely $3-5$-flowered. Flowers white or yellow with purplish brown spots, $2.5-3 \mathrm{~cm}$ across.

Habitat: Growing in shaded and humid places in dense forests, banks of streams. Voucher specimen: Gogoi et al. 0233. Flowering: June - July. Local distribution: Joypur R.F, Assam. General distribution: NE India, China, Indonesia, Malaysia, Nepal, Thailand, Vietnam. (Figure 3)

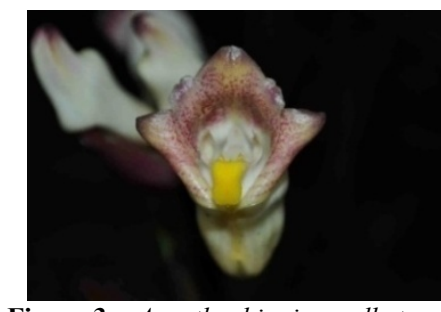

Figure 3. Acanthephippium sylhetense

\section{Aerides Loureiro}

About 20 species in the world, distributed from India to Vietnam, Malaysia and Japan, 10 species recorded in India, 5 species in North East India. (I. C. Barua:2001) and 2 species in Joypur Reserve Forest.

Aerides odorata Lour., Fl. Cochinch. 2: 525. 1790; Hook. f. Fl. Brit. India, 6:47, 1890; Chowdhery, Orch. Fl. Arunachal Prad. 49. 1998.

Plant $21-40 \mathrm{~cm}$ long. Stem $9-18 \mathrm{~cm}$ long, stout, pendent, branched, covered by persistent leaf sheaths. Leaves $10-19 \times 2.8-4.2 \mathrm{~cm}$, oblong, round-lobed at apex, incurved, distichous, jointed, pale green. Inflorescences $1-3$, pendent, racemose, densely many-flowered, $10-15 \mathrm{~cm}$ long, stout, glabrous. Flowers white with purple blotches at the 
extremities, $2.2-2.5 \mathrm{~cm}$ across, fragrant.

Habitat: Epiphyte in lowland forest. Voucher specimen: Gogoi et al.0156. Flowering : May-June. Local distribution: Joypur R. F, Assam. General distribution: India, Nepal, Bhutan, Burma, Andaman Is., Thailand, Vietnam, Peninsular Malaysia, Sumatra, Java, Borneo, Philippines. (Figure 4)

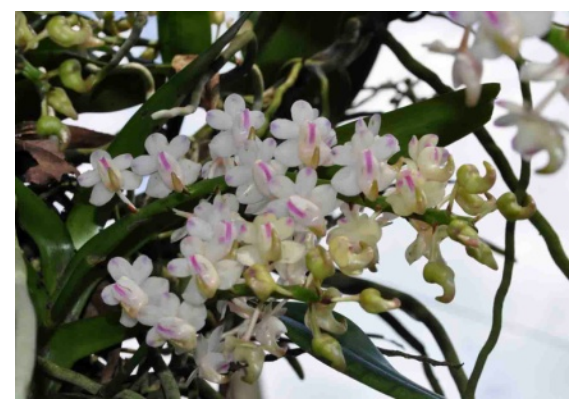

Figure 4. Aerides odorata

Aerides rosea Lodd. ex. Lindl. \& Paxt. Fl. Gard. 2: 109. 1852; Chowdhery, Orch. Fl. Arunachal Prad. 49. 1998; Mishra, Orch. India. 280. 2007.

Plant $25-35 \mathrm{~cm}$. stem stout, covered with sheaths. Leaves 13-33 $\times 2.4-4 \mathrm{~cm}$, oblong, 2-lobed, deeply channeled above, keeled beneath, curved, jointed. Inflorescence arising from peduncle sheath, racemes or paniculate, densely many-flow ered, $20-40 \mathrm{~cm}$ long, sheathed; sheaths 5-8 $\mathrm{mm}$ long, tubular, broadly ovate; rachis $21-31 \mathrm{~cm}$ long; floral bracts $3.5-5 \mathrm{~mm}$ long. Flowers fragrant, purplish-pink suffused with white, dark pink spotted, opening widely, about $2 \mathrm{~cm}$ across.

Habitat: Epiphyte in mixed deciduous forest and humid evergreen forest. Voucher specimen: Gogoi et al. 0131. Flowering: May. Local distribution: Joypur R. F, Assam. General distribution: N.E. India, Bhutan, Burma, China (Yunnan), Thailand, Vietnam.

\section{Agrostophyllum Blume}

About 4 species in India, 1 in Assam and 1 in Joypur Reserve Forest.

Agrostophyllum planicaule (Wall. ex Lindl.) Reichb. f. in W.G.Walpers, Ann. Bot. Syst. 6: 909. 1864. Agrostophyllum khasianum Griffith in Calcutta Jour. Nat. Hist. 4: 378, t. 19. 1844; Hook. f., Fl. Brit. India 5: 824. 1890; Chowdhery, Orch. Fl. Arunachal Prad. 53. 1998.

Plant $23-31 \mathrm{~cm}$ tall. Stems $7-10 \mathrm{~cm}$ long, fusiform, compressed, covered by overlapping sheaths. Leaves $7-14$. $5 \times 1.6-2.2 \mathrm{~cm}, 3$ or 4 , terminal, distichous, alternate, narrowl y ovate-oblong, tapering to each end, shortly petiolate, erect. Inflorescence $2-2.3 \mathrm{~cm}$ across, globose-capitate, densely many-flowered; peduncle short. Flowers white, 6-8 $\mathrm{mm}$ across.

Habitat: Epiphyte in mixed deciduous forest and humid evergreen forest. Voucher specimen: Gogoi et al. 0132. Flowering: August - October. Local distribution: Joypur R. F, Assam. General distribution: India, Nepal, Myanmar, Thailand, Cambodia and Vietnam.

\section{Anoectochilus Blume}

About 40 species known from India, S.E. Asia to Australia; 16 species in India, 3 species in Assam, 2 in Joypur Reserve
Forest.

Anoectochilus brevilabris Lindl. Gen. Sp. Orchid. 499. 1840; Hook. f., Fl. Brit. India 5: 824. 1890. Anoectochilus sikkimensis King \& Pantl., J. Asiat. Soc. Bengal, Pt. 2, Nat. Hist. 65: 124 (1896).

Plant $13-24 \mathrm{~cm}$ tall. Stem $3 \mathrm{~mm}$ wide, decumbent, glabrous. Leaves $4-6,3.4-6 \times 2.3-4 \mathrm{~cm}$, clustered at base of stem, petiolate, elliptic-ovate, acute, purple-green with yellow reticulations. Inflorescence densely many flowered, $7-12 \mathrm{~cm}$ long. Flowers up to $1.5-1.8 \mathrm{~cm}$ across; sepals olive-green and white, lip white, mesochile teeth green.

Habitat: Terrestrial in dense humid evergreen forest. Voucher specimen: Gogoi et al. 0312. Flowering: April June. Local distribution: Joypur R. F, Assam. General distribution: India (Assam), eastern Himalayas. (Figure 5)

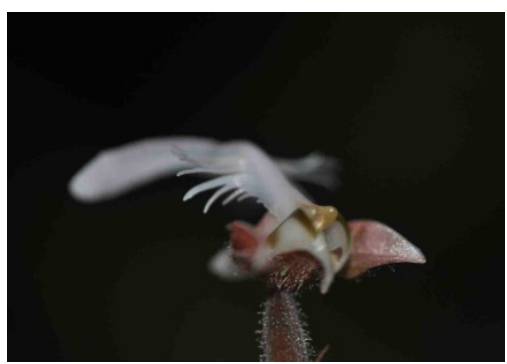

Figure 5. Anoectochilus brevilabris

Anoectochilus roxburghii (Wall.) Lindl. in J.F.Royle, III. Bot. Himal. Mts.: 368. 1839.

Plants up to $27 \mathrm{~cm}$ long. Leaves petiolate, $3-6 \times 2-5 \mathrm{~cm}$ wide, ovate to ovate-oblong, acute, in a basal rosette, velvety, purplish-red with golden reticulation. Inflorescence laxly many flowered, $13-17 \mathrm{~cm}$ long, glandular-pubescent, with 3 to 5 oblong sheathing bracts. Flowers up to $2 \mathrm{~cm}$ long, resupinate; sepals and petals pale pink-white, lip white.

Habitat: Terrestrial in dense humid evergreen forest. Voucher specimen: Gogoi et al. 0490. Flowering: JulyAugust. Local distribution: Joypur R. F, Assam. General distribution: N.E. India, Nepal, Bhutan, Burma, China, Thailand, Vietnam.

\section{Bulbophyllum Du. Petit Thouars}

About 1000 species in South East Asia, Africa, Australia and tropical America, about 100 species in India and 62 species are known from North Eastern region. Assam represents about 24 species among them 8 species grow in Joypur.

Bulbophyllum affine Lindl. Gen. Sp. Orchid. 48. 1830; Hook. f. Fl. Brit. India 5: 756. 1890; Chowdhery, Orch. Fl. Arunachal Prad. 87. 1998.

Pseudobulbs $2.6-4 \times 0.4-0.6 \mathrm{~cm}$, inserted at intervals of $3.7-7.5 \mathrm{~cm}$ along rhizome, erect, cylindric, rugose. Leaf $9-17 \times 2.5-3.3 \mathrm{~cm}$, solitary, coriaceous, linear-oblong to narrowly oblong-elliptic, apex obtusely and inconspicuously 2-lobed. Inflorescence basal, very short. Flowers white with pink lined, about $3 \mathrm{~cm}$ long, solitary.

Habitat: Epiphyte in mixed deciduous forest and humid evergreen forest. Voucher specimen: Gogoi et al. 0098. Flowering: June- August. Local distribution: Joypur R. F, 
Assam. General distribution: N.E. India, Bhutan, China, Thailand, Cambodia, Vietnam, Nepal. (Figure 6)

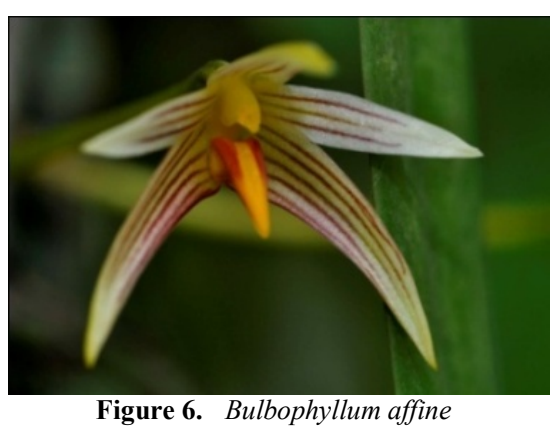

Bulbophyllum andersonii (Hook. f.) J.J. Smith in Bull. Jard. Bot. Buitenz. Ser. 2, 8: 22. 1912; Seidenf., Nots. Cirrhopetalum Lindl. 67. 1987; Chowdhery, Orch. Fl. Arunachal Prad. 92. 1998.

Cirrhopetalum andersonii Hook. f., Fl. Brit. India 5: 777. 1890.

Pseudobulbs $2-3.4 \times 0.4-0.6 \mathrm{~cm}$, ellipsoid to ovoid, smooth, fibrous at base. Leaf solitary, $8-14 \times 2-3.6 \mathrm{~cm}$, oblong to linear-oblong, apex subacute to emarginated, narrowed at base; petiole $2 \mathrm{~cm}$ long, channeled. Inflorescence basal from pseudobulb base, erect, umbellate, 4 to 9 -flowered. Flowers $1.5 \mathrm{~cm}$ long, sepals and petals whitish, densely spotted with purplish red; lip purple.

Habitat: Epiphyte in mixed deciduous forest and humid evergreen forest. Voucher specimen: Gogoi et al. 0112. Flowering: October. Local distribution: Joypur R. F, Assam. General distribution: N.E. India, China, Myanmar, Vietnam.

Bulbophyllum careyanum (Hook. f.) Spreng., Syst. Veg. 3: 732. 1826; Hook. f., Fl. Brit. India 5: 760. 1890; Barua, Orch. Fl. Kamrup dist. 144. 2001.

Pseudobulbs 3-6×2.8-3.4cm, globose-ovoid to narrowly ovoid, lightly grooved, inserted at intervals of $3.3-8.9 \mathrm{~cm}$ along rhizome. Leaf $11-23 \times 3.6-4.9 \mathrm{~cm}$ solitary, erect to suberect, oblong to linear-oblong, obtuse, sessile to shortly petiolate. Inflorescence lateral from pseudobub base, decurv ed, racemose, cylindric, densely many-flowered; peduncle $5-8 \mathrm{~cm}$ long, stout, sheathed. Flowers purplish-brown, $0.5-0.8 \mathrm{~cm}$ long.

Habitat: Epiphyte on tree trunks in dense humid evergreen forest. Voucher specimen: Borah et al. 0176. Flowering: October- December. Local distribution: Joypur R. F, Assam. General distribution: N.E. India, Nepal, Bhutan, Burma, Thailand, Vietnam.

Bulbophyllum delitescens Hance in Jour. Bot. 14: 44. 1867; Scidenf. Not. Cirrhopetalum Lindl. 215. 1987; Chowdhery, Orch. Fl. Arunachal Prad. 101. 1998.

Pseudobulb $1.5-3 \mathrm{~cm}$ long, $3-11 \mathrm{~cm}$ apart on rhizome, ovate or ovide-conical, covered, with long persistent sheaths. Leaves $15-25 \times 3-5 \mathrm{~cm}$, oblong-oblanceolate, leathery, obtuse at apex, petiolate, scapes from base of pseudobulb, erect, $10-22 \mathrm{~cm}$ long, with 3 tubular sterile bracts at long intervals. Inflorescence 3-8 flowered, sub-umbellate, Flowers reddish-brown.

Habitat: Epiphyte in dense humid evergreen forest near a waterfall. Voucher specimen: Gogoi et al. 0301. Flowering: June-July. Local distribution: Joypur R. F, Assam. General distribution: N.E. India, China, Vietnam.

Bulbophyllum ebulbum King \& Pantl. in Jour. Asiat. Soc. Bengal 64: 334. 1895 et in Ann. Roy. Bot. Gard. Calcutta 8: 83, t. 115. 1898; Chowdhery, Orch. Fl. Arunachal Prad. 104. 1998; Pradhan, Indian Orchid- II: 428. 1979.

Pseudobulb absent; rhizome slender to stout, $4-5 \mathrm{~mm}$ thick; roots arising from nodes. Leaf $14-22 \times 2.8-4 \mathrm{~cm}$, solitary, oblong-lanceolate, obtuse to subacute, inserted at intervals of $4-6 \mathrm{~cm}$ along rhizome; petiole $4.5-5.7 \mathrm{~cm}$ long, channeled. Inflorescence basal, close to leaf, racemose, arcuate, laxly many-flowered, $3.5-4.5 \mathrm{~cm}$ long, sheathed at base. Flowers pale-green, about $0.8 \mathrm{~cm}$ long.

Habitat: Epiphyte on tree trunks in dense humid evergreen forest. Voucher specimen: Gogoi et al. 0492. Flowering: May- June. Local distribution: Joypur R. F, Assam. General distribution: N.E. India, China, Thailand, Vietnam, Malaysia, Vietnam, Java, Sumatra, Borneo, Philippines.

Bulbophyllum odoratissimum (J.E. Smith) Lindl. Gen. Sp. Orchid. 55. 1830; Hook. f., Fl. Brit. India 5: 758. 1890; Chowdhery, Orch. Fl. Arunachal Prad. 123. 1998.

Pseudobulbs $1.8-2.4 \times 0.6-0.8 \mathrm{~cm}$, erect, cylindric, smooth to ridged, inserted at intervals of $2.3-4.7 \mathrm{~cm}$ along rhizome. Leaf 3.4-5×1.4-1.6cm, solitary, oblong-elliptic to oblonglanceolate, apex emarginated, subsessile to shortly petiole; petiole $1-3 \mathrm{~mm}$ long. Inflorescence 1 , basal from pseudobulb, compact umbel, densely 7-14 flowered, $3.5-8 \mathrm{~cm}$ long, sheathed. Flower 5-7mm long, white, fragrant.

Habitat: Epiphyte in dense humid evergreen forest near a waterfall. Voucher specimen: Gogoi et al. 0256. Flowering: May-September. Local distribution: Joypur R. F, Assam. General distribution: N.E. India, Nepal, Burma, China, Thailand, Vietnam.

Bulbophyllum protractum Hook. f. Fl. Brit. India, 5: 758. 1890; Pradhan, Indian Orchid- II: 409. 1979.

Pseudobulbs $1.5-2 \mathrm{~cm}$ long, about $3 \mathrm{~cm}$ apart on branched, obliquely narrowly cylindric. Leaves $4-5 \times 0.2-1.2 \mathrm{~cm}$, oblong, acute sessile; scape filiform, 2-3 flowered, slightly longer than pseudobulb, and arising from base of pseudobulb or from any point on the rhizome. Flowers $0.6 \mathrm{~cm}$ long, pale yellow.

Habitat: Growing epiphyte in evergreen forest. Voucher specimen: Gogoi et al, 0491. Flowering: June- July. Local distribution: Joypur R. F, Assam. General distribution: India and Burma.

Bulbophyllum roxburghii (Lindley) Reichenbach $f$. in W.G.Walpers, Ann. Bot. Syst. 6: 263. 1861; Cirrhopetalum roxburghii Lindley, Gen. Sp. Orchid. Pl.: 58. 1830; Bulbophyllum sikkimense (King \& Pantling) J.J.Smith, Bull. Jard. Bot. Buitenzorg, II, 8: 28. 1912.

Epiphyte. Pseudobulb, ovoid globose, $1-1.5 \mathrm{~cm}$ long. Leaves fleshy, oblanceolate, $7-8.8 \times 1-2 \mathrm{~cm}$. Inflorescence 6-8cm long umbellate, arising from base of pseudobulb; flowers small, dirty brown or pale rosy, spotted with purple.

Habitat: Growing epiphyte in evergreen forest. Voucher specimen: Gogoi et al, 0721. Flowering: April- July. Local 
distribution: Joypur R. F, Assam. General distribution: India (Assam) and Eastern Himalaya.

Bulbophyllum spathulatum (Rolfe ex. E. Cooper) Seidenf. In Bot. Tidsskr. 65: 347. 1970; Chowdhery, Orch. Fl. Arunachal Prad. 146. 1998.

Pseudobulbs 2-3x0.6-0.8cm, cylindric-ovoid, narrow, suberect to oblique. Leaf $4.5-7.5 \times 1.5-2 \mathrm{~cm}$, solitary, coriaceous, oblong-elliptic, obtuse to subacute. Inflorescenc e basal from pseudobulb, subumbellat, 5 to 9-flowered, 2-3cm long, sheathed. Flowers rose red, deeply spotted, 1.6 cm long.

Habitat: Epiphytic in riverine forest and in secondary evergreen forest. Voucher specimen: Gogoi et al, 0276. Flowering: April. Local distribution: Joypur R. F, Assam. General distribution: N.E. India, Burma, Thailand, Vietnam.

\section{Calanthe R. Brown}

About 150 species throughout the tropics and sub tropical Asia; 23 species in India, 9 in Assam, 2 species in Joypur Reserve Forest.

Calanthe lyroglossa Rchb. f., Otia Bot. Hamburg. 1: 53. 1878; Seidenfaden, Opera Botanica 114: 99. 1992; Mishra, Orch. India 285. 2007.

Plant 30-50cm tall; rhizome short, stout. Pseudobulbs 2-3 $\mathrm{cm}$ long, clavate-cylindric. Leaves $3-5,30-60 \times 3-8.5 \mathrm{~cm}$, basal; blade oblanceolate, plicate, glabrous, apex acuminate. Inflorescence arising from base of pseudobulb, erect, $30-50 \mathrm{~cm}$, stout, lower part with 3 or 4 tubular sheaths. Flowers small, yellow, turning black when dried; ovary and pedicel 10mm long, glabrous.

Habitat: Terrestrial in damp places on riversides. Voucher specimen: Gogoi et al, 0486. Flowering: NovemberFebruary. Local distribution: Joypur R. F, Assam. General distribution: India, China, Burma, Taiwan, Thailand, Cambodia, Vietnam, Peninsular Malaysia, Philippines.

Calanthe sylvatica (Thou.) Lindl., Gen. Sp. Orchid. Pl.: 250. 1833. Calanthe masuca (D. Don) Lindl., Gen. Sp. Orchid. 249. 1830; Hook. f., Fl. Brit. India 5: 850. 1890.

Plant $25-57 \mathrm{~cm}$ tall. Pseudobulbs $2-4 \mathrm{~cm}$, narrowly conical-cylindric, 4 or 5 noded. Leaves 4-6, 15-37×4-11 cm, large, broadly elliptic, acute to acuminate, petiolate. Inflores cence terminal, laxly few to many-flowered, $21-35 \mathrm{~cm}$ long, pubescent, sheathed. Flowers $2.5-4.2 \mathrm{~cm}$ across, pale pinkish purple with purple lip sometimes flushed with orange.

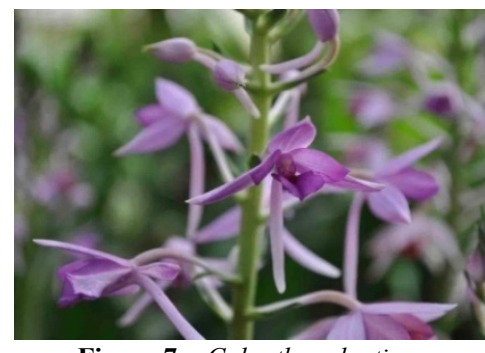

Figure 7. Calanthe sylvatica

Habitat: Terrestrial in damp places on riversides. Voucher specimen: Borah et al, 0161. Flowering: August - September. Local distribution: Joypur R. F, Assam. General distribution: India, China, Bhutan, Indonesia, Japan, Malaysia, Myanmar,
Nepal, Sri Lanka, Thailand, Vietnam, Africa, Madagascar. (Figure 7)

\section{Ceratostylis Blume}

About 60 species in India, S.E. Asia, Philippines and Pacific Islands. 1 species in India, Assam and Joypur Reserve Forest.

Ceratostylis sabulata B1., Bijdr. 7: 206. 1825; Chowdhery, Orch. Fl. Arunachal Prad. 167. 1998; Mishra, Orch. India 286. 2007.

Plant $13-24 \mathrm{~cm}$ tall, closely tufted, rush-like, sheathed at base, dark green. Leaf solitary, linear, subterete, grooved on one side, arising from stem apex, overtopping. Inflorescence, $1.5-2.7 \times 0.2-0.4 \mathrm{~cm}$, terminal, capitate, sessile, dense, $0.3-0.5 \mathrm{~cm}$ long. Flowers not opening widely, sparsely pubescent, sepals and petals yellow or dull red-purple, lip yellow.

Habitat: Epiphyte on tree trunk in dense humid evergreen forest. Voucher specimen: Gogoi et al, 0154. Flowering: May - August. Local distribution: Joypur R. F, Assam. General distribution: N.E. India, Bhutan, China, Thailand, Vietnam, Peninsular Malaysia, Sumatra, Java, Borneo, New Guinea, Philippines. (Figure 8)

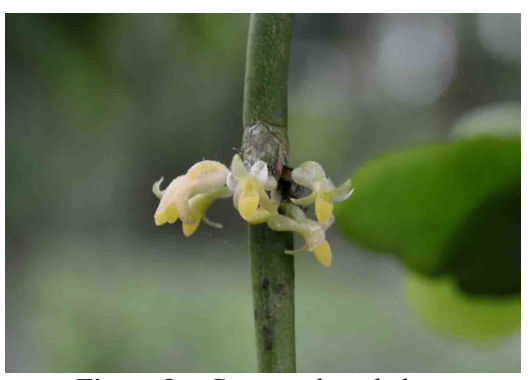

Figure 8. Ceratostylis sabulata

\section{Chrysoglossum Blume}

About 6 species in India, 3 in Assam, 2 species in Joypur Reserve Forest.

Chrysoglossum ornatum B1., Bjdr. 338. 1825. Chrysoglossum erraticum Hook. f. Fl. Brit. India 5: 784, 1890; Chowdhery, Orch. Fl. Arunachal Prad. 197. 1998.

Plant $26-41 \mathrm{~cm}$ tall. Pseudobulbs $3-5.4 \times 2-3 \mathrm{~cm}$, ovoid to tapering, terete. Leaf solitary, $15-30 \times 5-8 \mathrm{~cm}$, lanceolate, acuminate. Inflorescence 10-15-flowered, 13-22cm long, sheathed. Flowers 1.8-2cm long; sepals and petals green to yellow, lip white or yellowish with yellow.

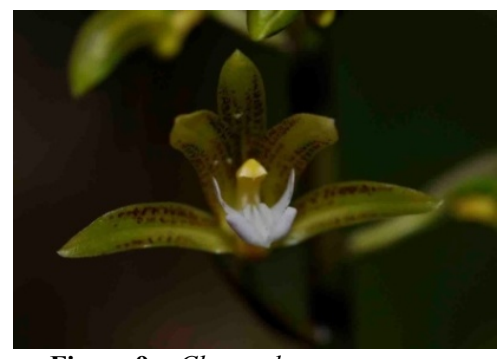

Figure 9. Chrysoglossum ornatum

Habitat: Shaded and humid places in forests. Voucher specimen: Borah et al, 0252. Flowering: August - October. Local distribution: Joypur R. F, Assam. General distribution: 
N.E. India, China, Taiwan, Bhutan, Cambodia, Indonesia, Malaysia, Nepal, Philippines, Sri Lanka, Thailand, Vietnam. (Figure 9)

Chrysoglossum robinsonii Ridley in Jour. Fed. Mal. St. Mus. 5: 157. 1915; Chowdhery, Orch. Fl. Arunachal Prad. 197. 1998; Mishra, Orch. India 287. 2007.

Plant rhizomatous; pseudobulbs $4 \times 0.2-0.4 \mathrm{~cm}$, cylindric, usually petiolate base slightly dilated; Leaf $7-15 \times 4-7 \mathrm{~cm}$, papery, base subrounded, acute. Inflorescence $14-18 \mathrm{~cm}$ long, glabrous, with 2-4 membranous tubular sheaths, laxly 4-7-flowered. Flowers medium- sized, sepals and petals green, lip white, column yellow.

Habitat: Shaded and humid places in dense forests. Voucher specimen: Gogoi et al, 0237. Flowering: June - July. Local distribution: Joypur R. F, Assam. General distribution: India, China, Taiwan, Thailand, Vietnam.

\section{Cleisocentron Bruhl}

1 species in India, Assam and Joypur Reserve Forest.

Cleisocentron pallens (Cath. ex Lindl.) N. Pearce \& P.J.Cribb, Edinburgh J. Bot. 58: 118. 2001. Cleisocentron trichromum (Reichb. f.) Bruhl, Guid. Orch. Sikkim 137. 1926; Chowdhery, Orch. Fl. Arunachal Prad. 199. 1998.

Plant $34-96 \mathrm{~cm}$ long. Stem branched, covered with fibrous leaf sheaths. Leaves many, 9-17×1.4-2cm, narrowly oblong, tapering slightly, apex obliquely bifid, sessile. Inflorescence divergent, second, few-flowered, $3.7-6.9 \mathrm{~cm}$ long, sheathed at base. Flowers $2.4-2.8 \mathrm{~cm}$ across; sepals and petals pale straw-coloured, lined with pale pink, spur pink, column white.

Habitat: Epiphytic on tree trunks evergreen forests. Voucher specimen: Borah et al, 0230. Flowering: June - July. Local distribution: Joypur R. F, Assam. General distribution: India (Assam, Arunachal Pradesh), Eastern Himalayas and Sikkim. (Figure 10)

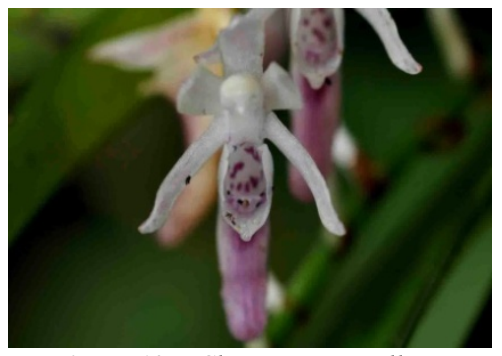

Figure 10. Cleisocentron pallens

\section{Cleisostoma Blume}

About 100 species distributed in India, South East Asia, Indonesia, New Guinea, Philippines and Pacific Island to Australia. About 19 species in India among them 7 species grow in Joypur.

Cleisostoma appendiculatum (Lindl.) Benth. \& Hook. f. ex Jackson in Index Kew. 1: 555. 1895; Chowdhery, Orch. Fl. Arunachal Prad. 200. 1998; Barua, Orch. Fl. Kamrup dist. 104. 2001.

Epiphyte; stem slender, erect, simple or branched, $20-40 \mathrm{~cm}$ long, coverd with leaf sheaths, rooting throughout the stem. Leaves fleshy, slightly curved, $7-10 \mathrm{~cm}$ long, spirally arranged, ascendant. Inflorescence few flowered, longer than leaves. Flowers $1.5 \mathrm{~cm}$ across, sepals and petals buff with purple longitudinal lines.

Habitat: Epiphytic on tree trunks in evergreen forests. Voucher specimen: Borah et al, 0230. Flowering: AugustOctober. Local distribution: Joypur R. F, Assam. General distribution: India, Myanmar. (Figure 11)

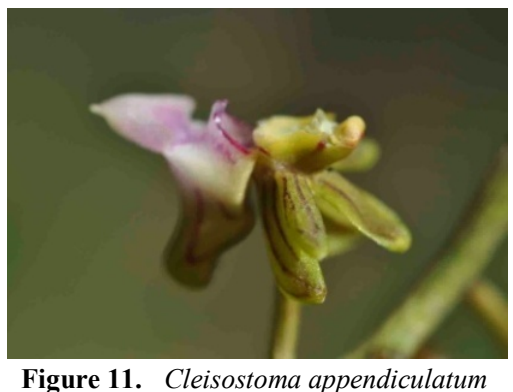

Cleisostoma filiforme (Lindl.) Garay in Bot. Mus. Leafl. Harvard Univ. 23(4): 171. 1972; Chowdhery, Orch. Fl. Arunachal Prad. 204. 1998; Pradhan, Indian Orchid-II: 509. 1979.

Plants epiphyte, pedulous, $40-100 \mathrm{~cm}$ long. Stem $2-3.5 \mathrm{~m}$ $\mathrm{m}$ wide, covered by leaf sheaths. Leaves $15-26 \times 0.2-0.3 \mathrm{~cm}$, terete, rugose, apex subacute, sessile. Inflorescence lateral, axillary, leaf-opposed, racemose, laxly 9 to 22-flowered, 9-11 cm long, glabrous, sheathed. Flowers about $1.3 \mathrm{~cm}$ across; sepals and petals yellowish green, with purplish brown stripes; lip white with purple-red midlobe, column yellow.

Habitat: Epiphytic on tree trunks in evergreen forests. Voucher specimen: Gogoi et al, 0494. Flowering: AprilJune. Local distribution: Joypur R. F, Assam. General distribution: N.E. India, Myanmar, Nepal, Thailand, Vietnam.

Cleisostoma linearilobatum (Seidenf. \& Smitinand) Garay, Bot. Reg. 31, mise. 59. 1845; Hook. f., Fl. Brit. India, 6: 75. 1890; Mishra, Orch. India, 288, 2007.

Plant $13-20 \mathrm{~cm}$ tall. Stem covered by leaf sheaths. Leaves $6-11.5 \times 1.5-2 \mathrm{~cm}$, distichously, apex unequally 2 -lobed, lobules rounded. Inflorescence $7-15 \mathrm{~cm}$ long, lateral, paniculate, laxly many-flowered. Flowers $3.8-5.5 \mathrm{~mm}$ across; Dorsal sepals and petals reddish-brown with intra-marginal bands of yellow, lip white with pink.

Habitat: Epiphytic on tree trunks in evergreen forests. Voucher specimen: Gogoi et al, 0493. Flowering: May-July. Local distribution: Joypur R. F, Assam. General distribution: N.E. India, Bhutan.

Cleisostoma paniculatum (Ker.-Gawl.) Garay in Bot. Mus. Leafl. Harvard Univ. 23(4): 173. 1972; Chowdhery, Orch. Fl. Arunachal Prad. 204. 1998; Mishra, Orch. India 288. 2007.

Plant erect, compressed terete, elongate, $30-60 \mathrm{~cm}$ long, sometimes branching, many leaved. Leaves $10-25 \times 0.8-2 \mathrm{~cm}$, distichous, flat, narrowly oblong or linear, leathery, unequally bilobed. Inflorescence axillary, much longer than leaves, many branched, many flowered. Flowers opening 
widely. Sepals and petals yellowish green abaxially, purplish brown adaxially, margins and midvein yellow. Lip yellow.

Habitat: Epiphytic on tree trunks in evergreen forests. Voucher specimen: Gogoi et al, 0707. Flowering: September-February. Local distribution: Joypur R. F, Assam. General distribution: India, Nepal, Bhutan, Burma, China (Yunnan), Thailand, Vietnam.

Cleisostoma racemiferum (Lindl.) Garay in Bot. Mus. Leafl. Harvard Univ. 23(4): 173. 1972; Chowdhery, Orch. Fl. Arunachal Prad. 204. 1998; Pradhan, Indian Orchid-II: 515. 1979.

Plant $18-27 \mathrm{~cm}$ tall. Stem covered by leaf sheaths, $0.8-1.2 \mathrm{~cm}$ wide. Leaves $5-9,15-30 \times 2.5-3.4 \mathrm{~cm}$, oblong, spreading, coriaceous, keeled beneath, apex unequally 2-lob ed, lobules broadly rounded, sessile, jointed. Inflorescence lateral, $14-18 \mathrm{~cm}$, leaf-opposite, paniculate, laxly many-flo wered. Flowers $0.7-0.8 \mathrm{~cm}$ across; sepals and petals brownish-black, edged with yellow, lip yellowish, column white.

Habitat: Epiphytic on tree trunks in evergreen forests. Voucher specimen: Gogoi et al, 0710. Flowering: JulySeptember. Local distribution: Joypur R. F, Assam. General distribution: India, Nepal, Bhutan, Burma, China (Yunnan), Thailand, Vietnam. Present ecological status: Endangered.

Cleisostoma simondii (Gagnep.) Scidenf. In Dansk Bot. Arkiv 29(3): 66. 1975; Chowdhery, Orch. Fl. Arunachal Prad. 208. 1998; Pradhan, Indian Orchid-II: 510. 1979.

Plant $20-38 \mathrm{~cm}$ tall. Stem erect, covered by leaf sheaths, $3-4 \mathrm{~mm}$ thick; sheaths overlapping. Leaves $5-8 \times 0.1-0.2 \mathrm{~cm}$, terete, fleshy, linear, apex subacute to obtuse, sessile, jointed. Inflorescence extra-axillary, pendent, long, simple or paniculate, laxly many flowered; $6-15 \mathrm{~cm}$ long, sheathed. Flowers $6.5-9 \mathrm{~mm}$ across; sepals and petals yellow to buff-coloured with many longitudinal purple to brown-purpl e markings, lip mid-lobe mauve to pink.

Habitat: Epiphyte on thick-barked tree trunks in evergreen and deciduous forest. Voucher specimen: Gogoi et al, 0221. Flowering: August- October. Local distribution: Joypur R. F, Assam. General distribution: India (N.E.), China, Thailand, Vietnam.

Cleisostoma subulatum B1., Bijdr. 363. 1825; Chowdhery, Orch. Fl. Arunachal Prad. 208. 1998; Pradhan, Indian Orchid-II: 514. 1979.

Plant pendulous. Stem $10-30 \mathrm{~cm}$ long, slender. Leaves $8.5-16 \times 0.8-1.1 \mathrm{~cm}$, distichous, fleshy, linear-oblong to oblo ng-lanceolate, acuminate, sheathed. Inflorescence lateral, leaf-opposed, pendent, racemose, laxly 7-18 flowered, $3-8 \mathrm{~cm}$ long. Flowers $5-7 \mathrm{~mm}$ across, scentless; sepals and petals pale yellow with intra-marginal bands of golden to red-brown, lip white with purple-pink apical lobe.

Habitat: Epiphyte on tree trunk in dense humid evergreen forest. Voucher specimen: Gogoi et al, 0097. Flowering: May-June. Local distribution: Joypur R. F, Assam. General distribution: Himalayas, India, Bangladesh, China, Taiwan, Yunnan, Cambodia, Philippines, Malaysia, Myanmar, Thailand, Vietnam, Java and Sumatra.

\section{Coelogyne Lindley}

43 species in India, 15 in Assam and 2 in Joypur.

Coelogyne fimbriata Lindl., Edwards's Bot. Reg. 11: t. 868. 1825; Hook. f., Fl. Brit. India 5: 836. 1890.

Plant $11-26 \mathrm{~cm}$ tall; rhizome creeping, sheathed. Pseudobulbs $2.8-7 \times 0.8-1.2 \mathrm{~cm}$, ovoid to ellipsoid, $3-4 \mathrm{~cm}$ apart on rhizome. Leaves $2,7.3-15 \times 1.5-2.5 \mathrm{~cm}$, oblong -elliptic, acute. Inflorescence hysteranthous, 1 to few -flowered, $3-8 \mathrm{~cm}$ long, zigzag. Flowers $3-4 \mathrm{~cm}$ across, paleyellow; sepals and petals yellowish. Lip yellowish, fimbriate at margins, keels 3 brownish; lip and column base brownish.

Habitat: Epiphyte on tree trunk in dense humid evergreen forest. Voucher specimen: Gogoi et al, 0709. Flowering: October- December. Local distribution: Joypur R. F, Assam. General distribution: NE India, Bhutan, Cambodia, Indonesia, Laos, NE Malaysia, Myanmar, Nepal, Thailand, Vietnam. (Figure 12)

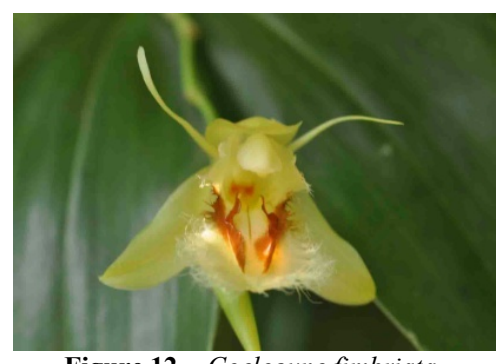

Figure 12. Coelogyne fimbriata

Coelogyne ovalis Lindl., Bot. Reg. 24: 91. Misc. 191. 1838; Hook. f. Fl. Brit. India 5: 836. 1890; Chowdhery, Orch. Fl. Arunachal Prad. 234. 1998.

Plants $14-21 \mathrm{~cm}$ tall, rhizome creeping, brancing, sheathed. Pseudobulbs $4.8-8.3 \times 1.5-1.9 \mathrm{~cm}$, ovoid-fusiform to fusiform, smooth, 4-6cm apart on rhizome, becoming ridged with age, sheathed at base. Leaves 2, 9-14×2.9-3.6cm, narrowly elliptic, acute to acuminate, petiolate. Inflorescence, 1 to few-flowered, $3-5 \mathrm{~cm}$ long. Flowers $4.6-5.5 \mathrm{~cm}$ across, pale yellowish, lip marked brown, keels darker brown, column yellowish-green.

Habitat: Epiphyte on tree trunk in dense humid evergreen forest. Voucher specimen: Gogoi et al, 0485. Flowering: August- December. Local distribution: Joypur R. F, Assam. General distribution: Epiphyte on tree trunk in dense humid evergreen forest.

Corymborkis Du. Petit Thouars

About 5 species in the world, 1 species in India, Assam and Joypur Reserve Forest.

Corymborkis veratrifolia (Reinw.) Bl., Coll. Orchid. Arch. Ind. 125, tt. 42 e \& 43. 1859; Pradhan, Indian Orchid-I: 100. 1979; Chowdhery, Orch. Fl. Arunachal Prad. 246. 1998.

Plant $75-90 \mathrm{~cm}$ tall. Stem terete, woody, leafy. Leaves $16-43 \times 4-13 \mathrm{~cm}$, plicate, elliptic to oblong-elliptic, acute to acuminate, sheathing at base. Inflorescence branched, erect or spreading, pendent, many-flowered. $9-13 \mathrm{~cm}$ long. Flowers fragrant, spreading, tubular, white, not widely spreading, $3.2-3.8 \mathrm{~cm}$ long.

Habitat: Terrestrial at shade and in dense humid evergreen forest with undergrowth of tall herbs. Voucher specimen: Gogoi et al, 0076. Flowering: March - May. Local 
distribution: Joypur R. F, Assam. General distribution: India, Thailand, Vietnam, tropical east Asia, Australia, Malaysia, Singapore, Sumatra, Java, Borneo, Moluccas, New Guinea, Philippines. (Figure 13)

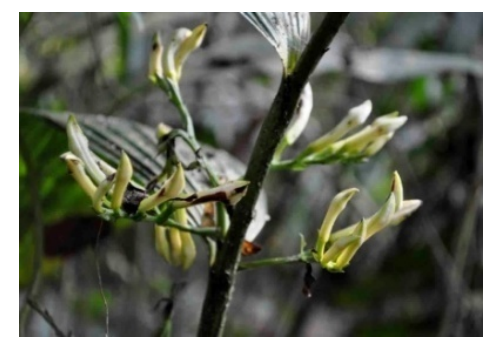

Figure 13. Corymborkis veratrifolia

\section{Cymbidium Swartz}

About 50 species distributed in S.E. Asia, China, Japan, Indonesia, India and Australia; 30 species in India, 12 in Assam and 3 species in Joypur Reserve Forest.

Cymbidium aloifolium (Lindl.) Sw. in Nova Acta Upsal. 2, 6: 73. 1799; Hook. f., Fl. Brit. India 6: 10. 1890; Chowdhery, Orch. Fl. Arunachal Prad. 253. 1998.

Plant $40-65 \mathrm{~cm}$ tall. Pseudobulbs $6-8 \times 3-4 \mathrm{~cm}$, inflated, ovoid, bilaterally flattened, enclosed in persistent leaf bases and sheaths. Leaves $4-5,34-65 \times 2.7-3.8 \mathrm{~cm}$, oblong, obtuse to emarginated, unequally bylobed at apex, coriaceous, arching. Inflorescence many flowered, pendulous, $50-70 \mathrm{~cm}$ long. Flowers $4-5 \mathrm{~cm}$ across, slightly fragrant; sepals and petals pale yellow to cream-yellow, with a broad central maroon-brown stripe; lip cream-colored.

Habitat: Epiphyte on tree trunk in dense humid evergreen forest. Voucher specimen: Borah et al, 0079. Flowering: April - May; Local distribution: Joypur R. F, Assam. General distribution: India, Sri Lanka, Nepal, Bangladesh, China, Burma, Thailand, Cambodia, Vietnam, Peninsular Malaysia, Java. (Figure 14)

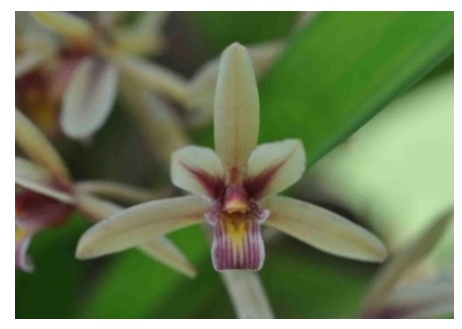

Figure 14. Cymbidium aloifolium

Cymbidium bicolor Lindl., Gen. Sp. Orchid. 164. 1833; Hook. f., Fl. Brit. India 6: 10. 1890.

Plant 24-58cm tall. Pseudobulbs narrowly ovoid, enclosed in persistent leaf bases; bladeless sheaths 4 or 5 . Leaves 5-6, 34-75×2.5-3.4cm, narrowly oblong, obtuse, stiff, arching; leaf base $3-12 \mathrm{~cm}$ long. Inflorescence pendulous, 11 to 20-flowered, 20-30cm long. Flowers 3-4 $\mathrm{cm}$ across; sepals and petals yellow with a central purplish red longitudinal stripe; lip cream-yellow.

Habitat: Epiphyte on tree trunk in dense humid evergreen forest. Voucher specimen: Gogoi et al, 0135. Flowering: May - June. Local distribution: Joypur R. F, Assam. General distribution: Thailand, Cambodia, Vietnam, Nepal, Bhutan,

\section{N.E. India, Burma, China.}

Cymbidium dayanum Reichb. f. in Gard. Chron. 710. 1869; Chowdhery, Orch. Fl. Arunachal Prad., 256, 1998; Mishra, Orch. India, 290, 2007.

Plant $27-83 \mathrm{~cm}$ tall. Pseudobulbs $3.7-4.5 \mathrm{~cm}$ long, fusiform, covered by scarious remains of leaf bases and bladeless sheaths. Leaves 5-8, 30-75×1-1.5cm, distichous, linear-elliptic, acute to acuminate, erect to arching, coriaceous. Inflorescence, pendulous, 8 to 13-flowered, $10-15 \mathrm{~cm}$ long. Flowers $4-5 \mathrm{~cm}$ across; sepals and petals white, with a central maroon stripe; lip maroon, tinged white at its base.

Habitat: Epiphyte on tree trunk in dense humid evergreen forest. Voucher specimen: Gogoi, 0116. Flowering: June July. Local distribution: Joypur R. F, Assam. General distribution: N.E. India, Burma, China, Taiwan, Japan, Thailand, Cambodia, Vietnam, Peninsular Malaysia, Sumatra, Borneo, Philippines.

\section{Dendrobium Swartz}

More than 1000 species and is widely distributed in Sri Lanka, India, China, Japan, Malaysia, Australia and Newzealand. In India it represents 102 species and nearly 20 species grow in Assam among them 13 species are grow in Joypur.

Dendrobium acinaciforme Roxb., Fl. Ind. 3: 487. 1832; Barua, Orch. Fl. Kamrup dist. 151. 2001; Chowdhery, Orch. Fl. Arunachal Prad. 283. 1998.

Plant 10-30cm long, compressed. Leaves $3.5-5 \times 0.6-0.8$ $\mathrm{cm}$, lanceolate, distichous, fleshy, enciform, acute, sessile, laterally compressed. Inflorescence racemose. Flowers solitary from axils of bracts, yellowish-white, small, about $8 \mathrm{~mm}$ across.

Habitat: Epiphyte on tree trunk in dense humid evergreen forest. Voucher specimen: Gogoi et al, 0117. Flowering: June - August. Local distribution: Joypur R. F, Assam. General distribution: India, China, Thailand, Cambodia, Vietnam and Malaysia.

Dendrobium aduncum Wall. In Bot. Reg. 1842, misc. 62, t. 15. 1846; Hook. f., Fl. Brit. India 5: 730. 1890; Chowdhery, Orch. Fl. Arunachal Prad. 283. 1998.

Plants pendulous, 30-60 cm long. Stems, branching, narrow, grooved; internodes sheathed, $1.8-4 \mathrm{~cm}$ long. Leaves $5.5-8.9 \times 0.8-1.6 \mathrm{~cm}$, oblong-lanceolate, acute to acuminate, minutely to obliquely emarginated, sessile. Inflorescence lateral, from leafless stem, 4-6 flowered, 3-8cm long, zigzag. Flowers $1.2-2 \mathrm{~cm}$ across, pale purple; sepals and petals pale pink; lip white; column white.

Habitat: Epiphyte on a small tree trunk in dense humid evergreen forest. Voucher specimen: Gogoi et al, 0078. Flowering: May. Local distribution: Joypur R. F, Assam. General distribution: N.E. India, Bhutan, Burma, China, Thailand, Vietnam.

Dendrobium aphyllum (Roxb.) Fischer in Gamble, Fl. Pres. Madras 1416. 1928; Hook. f., Fl. Brit. India 5: 738. 1890; Chowdhery, Orch. Fl. Arunachal Prad. 287. 1998.

Plans with pseudobulbs, $60-120 \mathrm{~cm}$ long, with a sub-orbicular bulbus base and tuft of basal roots, pendulous, 
leafless when flowering; inter nodes $3 \mathrm{~cm}$, striate, sheathed. Leaves deciduous, sessile, sheathing. Flowers, pale rose, 4.5-5 cm across; lip yellow.

Habitat: Epiphyte in mixed deciduous forest or open forest. Voucher specimen: Gogoi et al, 0077. Flowering: April May. Local distribution: Joypur R. F, Assam. General distribution: India, Nepal, Bhutan, Burma, China, Thailand, Cambodia, Vietnam, Peninsular Malaysia.

Dendrobium cathcartii Hook. f., Fl. Brit. India 5: 727. 1890, Chowdhery Orch. Fl. Arunachal Prad. 290. 1998.

Plant $40-66 \mathrm{~cm}$ tall. Stems erect, $0.4-0.5 \mathrm{~cm}$ thick, terete, uniform in size, many-leaved; internodes yellowish, sheathed, 2-2.4cm long. Leaves $8-12 \times 0.9-1.3 \mathrm{~cm}$, linear-lanceolate, obliquely notched, sessile, distichous, jointed. Inflorescence fasciculate, lateral, arising from nodes, 2-flowered. Flowers greenish-yellow, 2cm. across, fragrant; lip yellow with purple.

Habitat: Epiphyte on tree trunk in dense humid evergreen forest. Voucher specimen: Gogoi et al, 0148. Flowering: April - May. Local distribution: Joypur R. F, Assam. General distribution: India, Eastern Himalayas and Laos. Present ecological status: Endangered.

Dendrobium cumulatum Lindl. in Gard. Chron. 756. 1855; Hook. f., Fl. Brit. India 5: 731. 1890; Chowdhery, Orch. Fl. Arunachal Prad. 295. 1998.

Plant $28-54 \mathrm{~cm}$ tall. Stems erect, thickened upwards, internodes covered by leaf sheaths; internodes $2.5-3.6 \mathrm{~cm}$ long. Leaves $5-10 \times 1.5-3 \mathrm{~cm}$, oblong-lanceolate to elliptic, acute, distichous, sessile, sheathed. Inflorescencecorymbifor $\mathrm{m}$, borne on a leafless stem, 5 to 10 flowered, $2-4 \mathrm{~cm}$ long, glabrous, zigzag. Flowers white with purple; lip white suffused with yellow.

Habitat: Epiphyte on tree trunk in forest along a small stream. Voucher specimen: Gogoi, 0265. Flowering: March -May. Local distribution: Joypur R. F, Assam. General distribution: India, Nepal, Bhutan, Burma, Thailand, Cambodia, Vietnam, Borneo.

Dendrobium fimbriautm Hook., Exot. Fl. t. 71. 1823; Hook. f., Fl. Brit. India 5: 745. 1890; Chowdhery, Orch. Fl. Aunachal Prad. 301. 1998.

Plant $71-115 \mathrm{~cm}$ long. Stems erect or pendulous, tapering at end, yellowing, jointed, swollen at base, many-leaved; internodes $1.8-3 \times 0.7-2 \mathrm{~cm}$, sheathed. Leaves 9-14×1.5-2.7 $\mathrm{cm}$, oblong-lanceolate to narrowly elliptic-lanceolate, acute to acuminate, many-veined, deciduous, sessile, jointed with internode sheath. Inflorescences lateral, arising from nodes, pendulous, 5-15-flowered, 5-15cm long, glabrous. Flowers 4-5 cm across, sepals and petals golden yellow; lip golden yellow, with or without maroon blotch.

Habitat: Epiphyte in mixed deciduous forest, also in humid evergreen forest. Voucher specimen: Gogoi et al, 0101. Flowering: March - May. Local distribution: Joypur R. F, Assam. General distribution: India, Nepal, Bhutan, Burma, China, Thailand, Vietnam.

Dendrobium lituiflorum Lindl. in Gard. Chron. 372. 1856; Hook, Fl. Brit. India 5: 740. 1890; Chowdhery, Orch. Fl. Arunachal Prad. 308. 1998.
Plant pendulous, cylindric, up to $60-80 \mathrm{~cm}$ long, $7-10 \mathrm{~mm}$ in diam., slender, slightly fleshy, unbranched, with many nodes, internodes $3-3.5 \mathrm{~cm}$. Leaves $7.5-10 \times 1.6-2 \mathrm{~cm}$, narrowly oblong, linear-lanceolate, leathery, base sheathing, apex acuminate and slightly hooked on one side, deciduous, absent at the time of flowering. Inflorescences many, arising from old leafless stems, 2-5 flowered. Flowers 4-5 cm. across, pale purple inner surface of lip with a deep purple spot surrounded by a white circle.

Habitat: Epiphytic on tree trunks in open forests. Voucher specimen: Gogoi et al, 0057. Flowering: April - May. Local distribution: Joypur R. F, Assam. General distribution: N.E. India, Thailand, Vietnam, Burma, China.

Dendrobium moschatum (Buch.-Hum.) Sw. in Schrad., Neu. Jour. Bot. 1: 94. 1805; Barua, Orch. Fl. Kamrup Dist. 159. 2001; Chowdhery, Orch. Fl. Arunachal Prad. 311. 1998.

Plant $70-131 \mathrm{~cm}$ tall. Stems clustered, stout, terete, erect to pendulous, ridged, joined; internodes sheathed. Leaves $8-17 \times 2-4.5 \mathrm{~cm}$, lanceoate to elliptic-lanceolate, acute, subsessile, many veined, distichous, jointed. Inforescences lateral, arising from nodes of leafy or leafless stem, laxly 7-14 flowered, 10-18cm long. Flower orange yellow, 5-7cm across, deep yellow disk with a pale purplish brown blotch on either side.

Habitat: Epiphytic on tree trunks in open forests. Voucher specimen: Gogoi et al, 0058. Flowering: April - June. Local distribution: Joypur R. F, Assam. General distribution: India, Nepal, Bhutan, Burma, China, Thailand, Cambodia, Vietna $\mathrm{m}$.

Dendrobium nobile Lindl., Gen. Sp. Orchid. 79. 1830; Hook. f. Fl. Brit. India 5: 740. 1890; Chowdhery, Orch. Fl. Arunachal Prad. 314. 1998.

Plant forming large clumps. Stems $30-60 \mathrm{~cm}$ long, clustered, compressed, swollen at base, covered with sheaths, beaded, yellowish; internodes $2.8-3.5 \times 0.8-1.2 \mathrm{~cm}$. Leaves $6.5-12 \times 2.5-3 \mathrm{~cm}$, oblong, emarginated, many-veined, sessile, coriaceous, distichous, persistent. Inflorescences lateral, arising from nodes, 2-4-flowered, $1-1.5 \mathrm{~cm}$ long. Flowers $5-7 \mathrm{~cm}$. across, white merging with purple at apex; lip purple at tip, at middle followed by deep purple central blotch.

Habitat: Epiphyte in humid evergreen forest, also in the lowlands. Voucher specimen: Gogoi et al, 0346. Flowering: April - May. Local distribution: Joypur R. F, Assam. General distribution: India, Nepal, Bhutan, Burma, China, Taiwan, Thailand, Vietnam. (Figure 15)

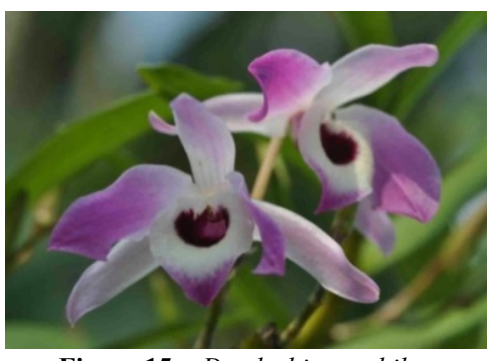

Figure 15. Dendrobium nobile

Dendrobium stuposum Lindl. in Bot. Reg. 52. 1838; Hook. f. Fl. Brit. India 6: 186. 1890; Chowdhery Orch. Fl. 
Arunachal Prad. 322. 1998.

Plant $13-34 \mathrm{~cm}$ tall. Stems caespitose, subclavate, grooved, sheathless; internodes $2.3-4.2 \mathrm{~cm}$ long. Leaves $3-7 \times 0.8-1.5$ $\mathrm{cm}$, oblong, obtuse-emarginate, distichous, sessile. Inflorescence lateral, arising from nodes, leaf-opposed or from leafless stem, 2 or 3-flowered. Flowers $1.6-2.1 \mathrm{~cm}$ across, sepals and petals white with purple margins, lip with a blotch of dull yellow-orange at apex.

Habitat: Epiphytic on tree trunks in open and mountain forests. Voucher specimen: Gogoi et al, 0504. Flowering: June- August. Local distribution: Joypur R. F, Assam. General distribution: India, Bhutan, Burma, China, Thailand.

Dendrobium sulcatum Lindl in Bot. Reg. n. 5. 24: t. 65. 1838; Hook. f. Fl. Brit. India 5: 749. 1890; Chowdhery, Orch. Fl. Arunachal Prad. 322. 1998.

Plant 19-42cm tall. Stem pseudobulbs, clavate, compressed, grooved, narrowed towards base, nodes several. Leaves 3 to $4,8-15 \times 3-5 \mathrm{~cm}$, subterminal, distichous, broaly elliptic to elliptic ovate, acute, sessile. Inflorescence $6-10 \mathrm{~cm}$ long, axilary, adjacent to leaf, subdensely 3 to 7-flowered. Flowers golden yellow, 2.5-3.5 cm across; lip with red-purple markings at base.

Habitat: Epiphytic on tree trunks in dense forests. Voucher specimen: Gogoi et al, 0153. Flowering: April-May. Local distribution: Joypur R. F, Assam. General distribution: N.E. India, Burma, China, Thailand.

Dendrobium terminale Par. \& Reichb. f. in Trans. Linn. Soc. 30: 149. 1859; Hook. f. Fl. Brit. India 5: 725. 1890; Chowdhry, Orch. Fl. Arunachal Prad. 193. 1998.

Plant $9-14 \mathrm{~cm}$ tall. Stem subclavate, slender at base, covered with leaf sheaths; internodes $0.8-1.1 \mathrm{~cm}$ long. Leav es 1.4-2×0.4-0.6cm, distichous, many, fleshy, oblong-ellipt ic, subacute to acute, equitant, jointed, base broad, sessile. Inflorescence terminal, 1 or 2-flowered. Flowers $1-1.2 \mathrm{~cm}$ long, white flushed pink.

Habitat: Epiphytic on tree trunks at forest. Voucher specimen: Borah et al, 0203. Flowering: April-June. Local distribution: Joypur R. F, Assam. General distribution: N.E. India, China, Malaysia, Myanmar, Thailand, Vietnam.

Dendrobium transparens Lindl., Gen. Sp. Orchid. 79. 1830; Hook. f. Fl. Brit. India 5: 738. 1890; Chowdhry, Orch. Fl. Arunachal Prad. 327. 1998.

Plant $30-54 \mathrm{~cm}$ tall, slender, erect, swollen at nodes, pseudobulbous at base; internodes covered by leaf sheaths, yellowing with age, $2.4-5.3 \mathrm{~cm}$. Leaves $7-11 \times 1.1-1.6 \mathrm{~cm}$, linear-lanceolate to oblong-elliptic, acute to oblique, sessile, jointed, many-veined. Inflorescence lateral, arising from leafless stem, 2-4-flowered. Flowers 4-5 cm across; sepals and petals white linged with purple at tips, lip white with large purple patch on the disc.

Habitat: Epiphytic on tree trunks. Voucher specimen: Gogoi et al, 0061. Flowering: April-May. Local distribution: Joypur R. F, Assam. General distribution: India, Nepal, Bhutan, Burma.

Didymoplexis Griffith

About 2 species in India, 1 in Assam and Joypur Reserve Forest.
Didymoplexis pallens Griff. in Calcutta Jour. Nat. Hist. 4: 383, t. 17. 1843; Hook. f., Fl. Brit. India 6: 122. 1890; Chowdhery, Orch. Fl. Arunachal Prad. 329. 1998.

Plant erect, leafless, slender, ridged, glabrous, distantly 1 to 3 -sheathed, $8-16 \mathrm{~cm}$ tall, pale buff-coloured, tubers fusiform, often moniliform, $1.8-3.5 \times 0.5-0.6 \mathrm{~cm}$. Inflorescen ce laxly 3-7-flowered. Flowers white, $0.8 \mathrm{~cm}$ across. Habitat: Terrestrial, growing in bamboo forest. Voucher specimen: Gogoi et al, 0198. Flowering: May-June. Local distribution: Joypur R. F, Assam. General distribution: N.E. India, N Fujian, Taiwan, Afghanistan, Bangladesh, Indonesia, Japan, Malaysia, New Guinea, Philippines, Thailand, Vietnam; Australia, SW Pacific islands. (Figure 16)

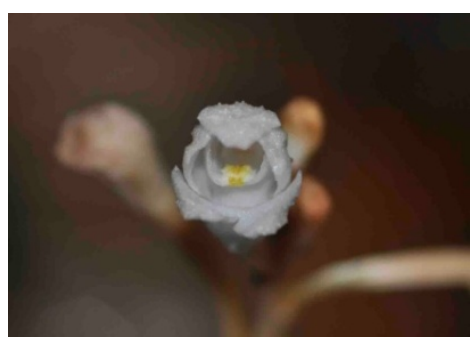

Figure 16. Didymoplexis pallens

Epipogium Gmelin ex Borkhausen

6 species in India, 1 in Assam and Joypur.

Epipogium roseum (D. Don) Lindl. in Jour. Linn. Soc. 1: 177. 1857; Chowdhery, Orch. Fl. Arunachal Prad. 343. 1998; Mishra, Orch. India 296. 2007.

Plant $13-46 \mathrm{~cm}$ tall; rhizome tuberous, internodes many. Stem $9-23 \mathrm{~cm}$ tall, erect, glabrous, sheathed. Inflorescence pale pink, laxly many-flowered, 5-20cm long, suberect to pendent. Flowers white with faint purple spots on lip.

Habitat: Terrestrial in evergreen forest. Voucher specimen: Gogoi et al, 0712. Flowering: October-December. Local distribution: Joypur R. F, Assam. General distribution: N.E. India, Tropical Africa, tropical continental Asia, Peninsular Malaysia, Sumatra, Java, Borneo, Sulawesi, Moluccas, New Guinea, Philippines, Australia, Vanuatu, New Caledonia.

Eria Lindley

Approximately 900 species, distributed in Si-lanka, India, Nepal, Myanmar, Malaya, Thiland, Combodia, Philippines, China, Japan to Java, Sumatra, Borneo, Celebes, New Guinea, Australia, And New Zealand. In India it represents 59 species and nearly 10 species in Assam among them Joypur Reserve Forest consist of 10 species of Eria.

Eria acervata Lindl. in Jour. Hort. Soc. 6: 57. 1851; Hook. f., Fl. Brit. India 5: 57. 1851; Chowdhery, Orch. Fl. Arunachal Prad. 350. 1998.

Pseudobulbs $3.5-6 \times 1-2 \mathrm{~cm}$, compressed, flask-shaped to ellipsoid, stacked to clustered, furrowed, sheathed when young. Leaves $4-7,6-15 \times 1.8-2.5 \mathrm{~cm}$, narrowly oblong to oblanceolate-oblong, obtuse-emarginate, tapered towards base, petiolate. Inflorescence $2-5,6-15 \mathrm{~cm}$ long, laxly many flowered. Flowers white, about $1.5 \mathrm{~cm}$ across.

Habitat: Epiphyte in dense humid evergreen forest. Voucher specimen: Gogoi et al, 0501. Flowering: May- July. Local distribution: Joypur R. F, Assam. General distribution: 
N.E. India, Nepal, Bhutan, Burma, Tibet, China, Thailand, Cambodia, Vietnam.

Eria amica Reichb. f., Xen. Orchid. 2: 162, t. 168. f. 6-9. 1870; Hook. f., Fl. Brit. India 5: 800. 1890; Chowdhery, Orch. Fl. Arunachal Prad. 350. 1998.

Plant $13-28 \mathrm{~cm}$ tall. Pseudobulbs $5-14 \times 0.8-1.5 \mathrm{~cm}$, superposed, erect-cylindric, sheathed at base. Leaves 2, $11-14 \times 1.7-2.1 \mathrm{~cm}$, arising from pseudobulb apex, linear-lanceolate to oblong-lanceolate, acute, tapered to petiolate base. Inflorescence arising from axils of leaf-sheaths, 1-3 from each pseudobulb apex, erect to suberect, laxly many flowered, $5-10 \mathrm{~cm}$ long, pubescent. Flowers pale-yellow, with reddish- brown nerves, about 1.2 cm across.

Habitat: Epiphyte in dense humid evergreen forest. Voucher specimen: Gogoi et al, 0482. Flowering: MarchMay. Local distribution: Joypur R. F, Assam. General distribution: N.E. India, Bhutan, Burma, China, Taiwan, Thailand, Cambodia, Vietnam.

Eria connata Joseph, Hegde \& Abbareddy in Bull. Bot. Surv. India 24: 114, f. 1-7. 1982; Chowdhery, Orch. Fl. Arunachal Prad. 361. 1998; Mishra, Orch. India 297. 2007.

Plant up to $25 \mathrm{~cm}$ long including leaves; roots wiry; Pseudobulbs $5-15 \times 0.8-1 \mathrm{~cm}$, subcylindric. Leaves 4 or 5 , $6-8 \times 1.2-1.8 \mathrm{~cm}$, narrowly elliptic, subfalcate, acuminate, distichously, sessile. Inflorescence axillary or leaf-opposed, pendent, racemose, globose to subglobose, proteranthous, densely many-flowered. Flowers fragrant; sepals and petals white, lip yellow.

Habitat: Epiphyte in dense humid evergreen forest. Voucher specimen: Gogoi et al, 0480. Flowering: JulySeptember. Local distribution: Joypur R. F, Assam. General distribution: N.E. India and Bhutan.

Eria ferruginea Lindl. in Bot. Reg. n.s. 25: t. 35. 1839; Hook. f., Fl. Brit. India 5: 804. 1890; Chowdhery, Orch. Fl. Arunachal Prad. 363. 1998.

Plant $19-37 \mathrm{~cm}$ tall. Pseudobulbs stem-like, cylindric, internodes 4 or 5 , branched, sheathed, $7-9 \mathrm{~cm}$ long. Leaves 2 , $11-16 \times 1.7-3 \mathrm{~cm}$, arising from pseudobulb apex, narrowly oblong-elliptic, to elliptic, acute to subacuminate, coriaceous, petiolate, jointed. Inflorescence arising from pseudobulb base, racemose, laxly 6-11-flowered, $7-10 \mathrm{~cm}$ long, toment ose, sheathed. Flowers $2.4-3 \mathrm{~cm}$, yellowish-green, washed with purple-pink, lip white with red-purple spotted.

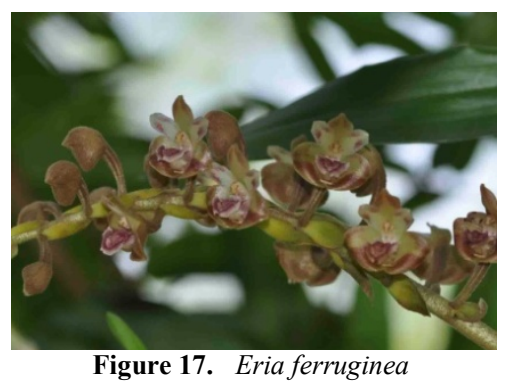

Habitat: Epiphyte in dense humid evergreen forest. Voucher specimen: Gogoi et al, 0507. Flowering: June- July. Local distribution: Joypur R. F, Assam. General distribution:

\section{N.E. India and Bhutan. (Figure 17)}

Eria lasiopetala (Willd.) Ormerod, Opera Bot. 124: 22. 1995. Eria pubescens (Hook. f.) Lindl. in Jour. Linn. Soc. 3: 54. 1858; Chowdhery, Orch. Fl. Arunachal Prad. 373. 1998; Barua, Orch. Fl. Kamrup Dist. 138. 2001.

Plant $19-33 \mathrm{~cm}$ tall; rhizome woody, sheathed. Pseudobulbs $3.6-5 \times 2.3-3.5 \mathrm{~cm}$, borne at intervals of $3-5 \mathrm{~cm}$ along rhizome, fusiform-ellipsoid, laterally compressed, furrowed, basally sheathed. Leaves $2-5,7-14 \times 2-3.5 \mathrm{~cm}$, lanceolate-oblong, acute to acuminate, petiolate. Inflorescence lateral, arising from pseudobulb base, erect, racemose, subdensely 6 to 13 -flowered, $7-12 \mathrm{~cm}$ long, softly white-tomentose. Flowers fragrant, $1.8-2.2 \mathrm{~cm}$ across, green to yellow, lip with crimson-purple.

Habitat: Epiphyte in humid evergreen forest, mixed deciduous forest. Voucher specimen: Gogoi et al, 0065. Flowering: March-April. Local distribution: Joypur R. F, Assam. General distribution: India, Himalayas, China, Taiwan, Yunnan, Cambodia, Indonesia, Laos, Malaysia, Myanmar, Thailand, Vietnam, Java and Sumatra.

Eria paniculata Lindl. in Wall., Pl. Asiat. Rar. 1: 32, t. 36. 1830; Hook. f., Fl. Brit. India 5: 789. 1890; Chowdhery, Orch. Fl. Arunachal Prad. 373. 1998.

Plant $30-44 \mathrm{~cm}$ tall. Stems caespitose, pendent, woody, covered in sheaths, $0.5-0.6 \mathrm{~cm}$ thick. Leaves many, $10-25 \times 1-1.5 \mathrm{~cm}$, linear-oblong, acuminate, fleshy, subsessil e to shortly petiolate. Inflorescence $2-4$, terminal, fasciculate to paniculate, densely many-flowered, $8-10 \mathrm{~cm}$ long. Flowers $0.8-1.1 \mathrm{~cm}$ across, acrid-scented; sepals pale yellowish-green, petals, lip and column with brownish- purple blotches.

Habitat: Epiphyte in dense humid evergreen forest. Voucher specimen: Gogoi et al, 0225. Flowering: DecemberMarch. Local distribution: Joypur R. F, Assam. General distribution: N.E. India, Nepal, Bhutan, Bangladesh, Burma, Thailand, Cambodia, Vietnam.

Eria pannea Lindl. in Bot. Reg. 64. misc. 79. 1842; Hook. f. Fl. Brit. India 5: 804. 1890; Chowdhery, Orch. Fl. Arunachal Prad. 373. 1998.

Plant $11-16 \mathrm{~cm}$ tall. Rhizome slender, covered by hairy sheaths. Leaves $3-6,4-17 \times 0.3-0.5 \mathrm{~cm}$, linear, laterally flattened, fleshy, subterete. Inflorescence arising amidst from leaves, 2 to 5 -flowered. $2-3 \mathrm{~cm}$ long, weakly zigzag, densely woolly. Flowers $1-2 \mathrm{~cm}$ wide, fragrant, pale yellow-green, lip dark purplish.

Habitat: Epiphyte in dense evergreen forest. Voucher specimen: Gogoi et al, 0147. Flowering: May-July. Local distribution: Joypur R. F, Assam. General distribution: N.E. India, Burma, China, Thailand, Cambodia, Vietnam, Peninsular Malaysia, Singapore, Sumatra, Borneo.

Eria pudica Ridl. in Jour. Linn. Soc. 32: 294. 1896; Chowdhery, Orch. Fl. Arunachal Prad. 377. 1998; Mishra, Orch. India 298. 2007.

Plant erect, pseudobulbs $2.5-3.5 \times 1-2 \mathrm{~cm}$, crowded, ovate, fusiform, $3-4 \times 0.5-0.7 \mathrm{~cm}$. Leaves solitary, $12-15 \times 3-3.5 \mathrm{~cm}$, elliptic, oblong, acute, petiolate. Inflorescence lateral, arising from the base of the pseudobulb, 3-4cm long, densely grayish white pubescent, maney flowered. Flowers 
spreading, pinkish white, pubescent, buff with darker Strips.

Habitat: Epiphyte in deciduous forest and evergreen forest. Voucher specimen: Gogoi et al, 0495. Flowering: AprilAugust. Local distribution: Joypur R. F, Assam. General distribution: N.E. India, Peninsular Malaysia and Borneo.

Eria pumila Lindl. Gen. sp. Orchid 68. 1830; Hook. f. Fl. Brit. India 5: 791. 1890.

Plant 11-16cm tall; rhizomatus, covered with fibrous sheaths. Pseudobulbs $4-6 \mathrm{~cm}$ long, crowded, subcylindric to clavate, compressed, weakly falcate, sheathed. Leaves 3 or 5 , 4.4-10.6×1-2cm, linear-lanceolate, acute, tapered to a short, sheathing petiole, jointed. Inflorescence short, globose-capit ate, densely many-flowered, $1-1.3 \mathrm{~cm}$ across, $2-4 \mathrm{~mm}$ long. Flowers $4-5 \mathrm{~mm}$ across, white flushed with pink, lip lateral lobes and column apex red.

Habitat: Epiphyte in humid evergreen forest, mixed deciduous forest. Flowering: January- March. Voucher specimen: Gogoi et al, 0502. Local distribution: Joypur R. F, Assam. General distribution: N.E. India, Bhutan and Myanmar.

Eria tomentosa (Koen.) Hook. f., Fl. Brit. India 5: 803. 1890; Chowdhery, Orch. Fl. Arunachal Prad. 385. 1998.

Plant rhizomatus, up to $15 \mathrm{~cm}$ long including leaves; rhizomes woody with nodal scars. Pseudobulbs $2.5-5 \times 1.5-2$ $\mathrm{cm}, 4-5 \mathrm{~cm}$ apart, ovate- oblong. Leaves $1-3,7-12 \times 1.5-2 \mathrm{c}$ $\mathrm{m}$, obovate- lanceolate, narrowed at base, acute. Inflorescence 10 to 15 -flowered, tomentose racemes. Flowers about $1.5 \mathrm{~cm}$ across, orange- yellow.

Habitat: Epiphyte in humid evergreen forest, mixed deciduous forest. Voucher specimen: Gogoi et al, 0503. Flowering: September- November. Local distribution: Joypur R. F, Assam. General distribution: N.E. India, Burma, Thailand, Vietnam.

Flickingeria Hawkes

About 60 species in S.E. Asia, Malaya, Archipelago, Pacific Islands and N. E. Australia; 7 species in India, 2 in Assam and 1 in Joypur.

Flickingeria fugax (Reichb. f.) Seidenf. in Dansk Bot. Arkiv 34: 46. 1980; Kumar et. Monilal, Cat. Ind. Orch. 74. 1994; Chowdhery, Orch. Fl. Arunachal Prad. 399. 1998.

Plants reect or pendulouus. Stems long, branched; internodes $1.7-2.8 \mathrm{~cm}$ long; pseudobulbs $3.4-5.8 \times 0.8-1 \mathrm{~cm}$, borne on stem nodes, narrowly fusiform, compressed, shining, grooved. Leaf solitary, $9-17 \times 2-2.5 \mathrm{~cm}$, terminal from pseudobulbs, linear-oblong to narrowly oblong-elliptic, minutely emarginated, veined, sessile. Inflorescences adaxial, 1 or 2 -flowered, short. Flowers $0.8-1.5 \mathrm{~cm}$ across, white, $3-3.5 \mathrm{~cm}$ across, fugacious.

Habitat: Epiphyte in humid evergreen forest, mixed deciduous forest. Voucher specimen: Borah et al, 0067. Flowering: March - October. Local distribution: Joypur R. F, Assam. General distribution: N.E. India, China, Bhutan, Myanmar, Thailand and Vietnam. (Figure 18)

\section{Gastrochilus David Don}

About 20 species in E. Asia, India, Malaysia and adjoining Islands; 12 species in India, 3 in Assam and Joypur.

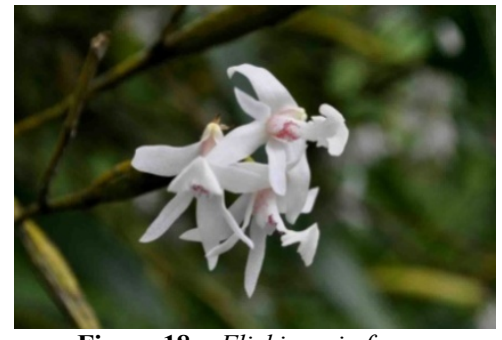

Figure 18. Flickingeria fugax

Gastrochilus calceolaris (Buch.-Ham. ex. J.E. Smith) D. Don, Prodr. Fl. Nepal. 32. 1852; Kumar et. Monilal, Cat. Ind. Orch. 75. 1994; Chowdhery, Orch. Fl. Arunachal Prad. 413. 1998.

Plant pendent, 9-22 cm long, sheathed. Leaves 3-7, 8-20×1.7-3.2cm, distichous, expetiolate, strongly falcate, linear-lanceolate to oblong-lanceolate. Inflorescence short, corymbose to subracemose, much shorter than the leaves, pendent, subdensely $6-8$ flowered. Flowers widely opening; sepals and petals yellow, with purplish brown markings; lip white with purple-red marks.

Habitat: Epiphyte in humid evergreen forest, mixed deciduous forest. Voucher specimen: Gogoi et al, 0320. Flowering: October - November. Local distribution: Joypur R. F, Assam. General distribution: N.E. India, China, Bhutan, Malaysia, Myanmar, Nepal, Thailand, Vietnam.

Gastrochilus dasypogon (J.E. Smith) O. Ktze., Rev. Gen. Pl. 2: 661. 1891; Kumar et. Monilal, Cat. Ind. Orch. 75. 1994; Chowdhery, Orch. Fl. Arunachal Prad. 4131998.

Plant 9-16cm tall. Leaves 4-5, 8-18×2.6-3.4cm, alternate, broadly ovate, fleshy, smooth above, minutely scaly beneath, apex acute, unequally 3-toothed, margins revolute, base sheathing. Inflorescence $3-4 \mathrm{~cm}$ long, corymbose to umbella te, densely 4-11-flowered. Flowers yellow with brownish -purple spots, $1.5-2 \mathrm{~cm}$ across.

Habitat: Epiphyte in humid evergreen forest, mixed deciduous forest. Voucher specimen: Gogoi et al, 0130. Flowering: October-November. Local distribution: Joypur R. F, Assam. General distribution: India, Nepal, Bhutan, Burma, Thailand and Vietnam. (Figure 19)

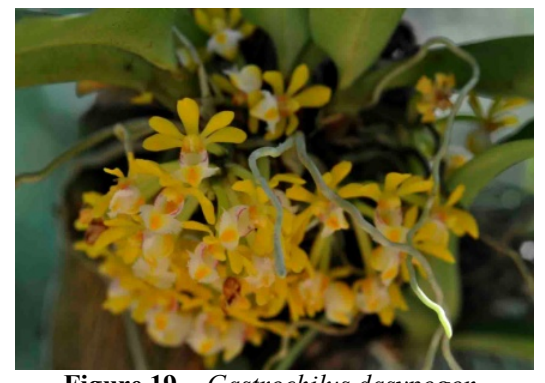

Figure 19. Gastrochilus dasypogon

Gastrochilus inconspicuus (Hook. f.) O. Ktze., Rev. Gen. Pl. 2: 661. 1891; Kumar et. Monilal, Cat. Ind. Orch. 75. 1994; Chowdhery, Orch. Fl. Arunachal Prad. 413. 1998.

Plant $10-30 \mathrm{~cm}$ tall. Stem terete, flexuous, branched, covered by leaf sheaths, red-brown, $3.5-15 \mathrm{~cm}$ long. Leaves $2.8-8 \times 0.1-0.2 \mathrm{~cm}$, terete, straight to weakly curved, 
rugose-straiate, dark green, sessile. Inflorescence lateral, racemose, 4-6 flowered, short, sheathed. Flowers white or yellowish-green, $0.5 \mathrm{~cm}$ across.

Habitat: Epiphyte in humid evergreen forest, mixed deciduous forest. Voucher specimen: Borah et al, 0070. Flowering: June - July. Local distribution: Joypur R. F, Assam. General distribution: India, Himalayas, Bangladesh and Nepal.

Geodorum G. Jackson

About 10 species in India, S.E. Asia, Australia, and S.W. Pacific Islands; 1 species in India, Assam and Joypur.

Geodorum densiflorum (Lamk.) Schltr. in Fedde Report. Behi. 4: 259. 1919; Kumar et. Monilal, Cat. Ind. Orch. 75. 1994; Chowdhery, Orch. Fl. Arunachal Prad. 418. 1998.

Plant $30-40 \mathrm{~cm}$ tall. Pseudobulb $2-4 \times 3-3.5 \mathrm{~cm}$, tuberous, subglobose, enclosed by scarious sheaths. Leaves 3-4, $10-31 \times 5-9 \mathrm{~cm}$, fasciculate, plicate, suberect, lanceolate, lanceolate-elliptic, or oblong-elliptic, acute to acuminate, petiolate. Inflorescence usually shorter than the leaves, compact, subglobose, pendent, 10-14-flowered, $16-30 \mathrm{~cm}$ long, sheathed. Flowers white to pinkish, lip with a central yellow callus and crimson-purple venation and streaks.

Habitat: Terrestrial in evergreen forest, also in grass land. Voucher specimen: Gogoi et al, 0238. Flowering: June - July. Local distribution: Joypur R. F, Assam. General distribution: India, Sri Lanka, China, Taiwan, Yunnan, Cambodia, Indonesia, Japan, Laos, Malaysia, Myanmar, New Guinea, Thailand, Vietnam; Australia. (Figure 20)

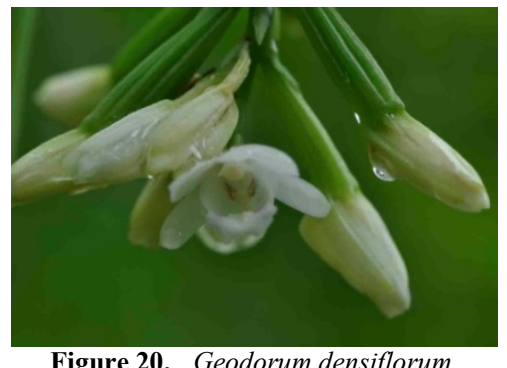

Figure 20. Geodorum densiflorum

\section{Goodyera Robert Brown}

About 40 species widely distributed in Mexico, S. E. Asia, Pacific Islands, New Guinea, Australia and Madagascar; 17 species in India, 4 in Assam, 1 species in Joypur.

Goodyera procera (Wall. ex Ker.-Gawl.) Hook., Exot. Fl. 1, 3: t. 39. 1823; Hook.f., Fl. Brit. India 6: 111. 1890; Chowdhery, Orch. Fl. Arunachal Prad. 431. 1998.

Plant $27-63 \mathrm{~cm}$ tall, leafy in the basal third, bearing 3 or 4 sheaths above. Leaves $7-14 \times 3.7-6 \mathrm{~cm}$, linear-lanceolate to ovate-lanceolate, petiolate, acuminate. Inflorescence densely many-flowered, $7-19 \mathrm{~cm}$ long, pubescent. Flowers fragrant, opening weakly, white tinged pale green.

Habitat: Terrestrial at the bank of a small stream and in evergreen forest, also on rock in a stream. Voucher specimen: Borah et al, 0205. Flowering: April- June. Local distribution: Joypur R. F, Assam. General distribution: India, Sri Lanka, Nepal, Bhutan, Burma, China, Taiwan, Japan, Thailand, Cambodia, Vietnam, Peninsular Malaysia, Sumatra, Java, Borneo, Philippines. (Figure 21)

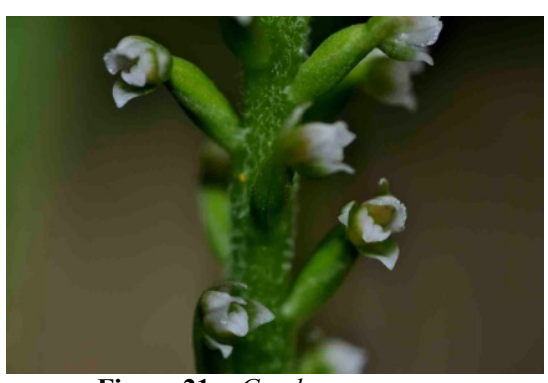

Figure 21. Goodyera procera

Hetaeria Blume

6 species in India, 2 in Assam and 1 in Joypur.

Hetaeria affinis (Griff.) Seidenf. \& Ormerod, Oasis, Suppl. 2: 9, 2001; Mishra, Orch. India, 304, 2007.

Plant $25-38 \mathrm{~cm}$ tall. Stem leafy, glabrous. Leaves $7.5-12 \times$ $4-5 \mathrm{~cm}$, ovate-elliptic, more or less oblique to subacute, 5 -veined. Inflorescence densely many-flowered, $7-14 \mathrm{~cm}$ long, densely glandular-pubescent. Flowers opening slightly, 5-6 $\mathrm{mm}$ across, green, tipped with pink; lip white.

Habitat: Terrestrial in secondary evergreen forest close to a small stream. Voucher specimen: Gogoi et al, 0505. Flowering: April- May. Local distribution: Joypur R. F, Assam. General distribution: N.E. India, Bhutan, Burma, China, Thailand, Peninsular Malaysia, Philippines.

Kingidium P.F. Hunt

About 5 species distributed in India, Sri Lanka, Myanmar, Thailand, Malaya, Indonesia and Philippines; 2 species India and Assam, 1 in Joypur.

Kingidium deliciosum (Reichb. f.) Sw. in Amer. Orchid Soc. Bull. 39: 1095. 1970; Mishra, Orch. India 304. 2007; Chowdhery, Orch. Fl. Arunachal Prad. 463. 1998.

Plant forming clumps, stem short, $0.5-1 \times 0.5-0.9 \mathrm{~cm}$, tufted. Leaves 3 or 4, obovate-oblong, obtuse to subacute, margins undulate, base narrowed, sessile, jointed, $7-16 \times 2.5-4.8 \mathrm{~cm}$. Inflorescence basal, one to several, paniculate or racemose, 5-9 flowered, $10-15 \mathrm{~cm}$ long, glabrous. Flowers greenish yellow; lip purple spoted.

Habitat: Epiphyte in gallery forest along a large river, in a shady spot in humid evergreen forest. Voucher specimen: Gogoi et al, 0210. Flowering: May- July. Local distribution: Joypur R. F, Assam. General distribution: N.E. India, Vietnam, Peninsular Malaysia, Sumatra, Java, Borneo, Sulawesi, Philippines. (Figure 22)

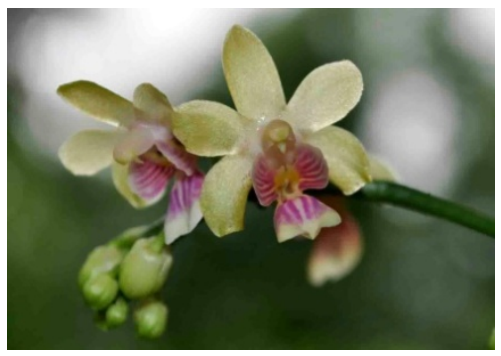

Figure 22. Kingidium deliciosum

Liparis L.C. Richard

About 250 species distributed mainly in the tropics of the world and comparatively less frequent in the temperate 
region; 45 species in India, 9 in Assam and 2 in Joypur.

Liparis mannii Reichb. f. in Flora 55: 275. 1872; Hook. f., Fl. Brit. India 5: 701. 1890; Chowdhery, Orch. Fl. Arunachal Prad. 479. 1998.

Plant $19-28 \mathrm{~cm}$ tall. Pseudobulbs $1.5-3 \times 0.6-0.9 \mathrm{~cm}$, crowded, narrowly ovoid, enveloped in ovate-lanceolate, acute, scarious sheaths. Leaf 9-21 $\times 0.7-1 \mathrm{~cm}$, solitary, arising from apex of pseudobulb, linear-oblong, acute, narrowed to a convolute. Inflorescence densely many-flowered, $5-14 \mathrm{~cm}$ long. Flowers yellowish, $5 \mathrm{~mm}$ across.

Habitat: Epiphyte in humid evergreen forest. Voucher specimen: Gogoi et al, 0124. Flowering: November January. Local distribution: Joypur R. F, Assam. General distribution: N.E. India, S Yunnan, Vietnam. (Figure 23)

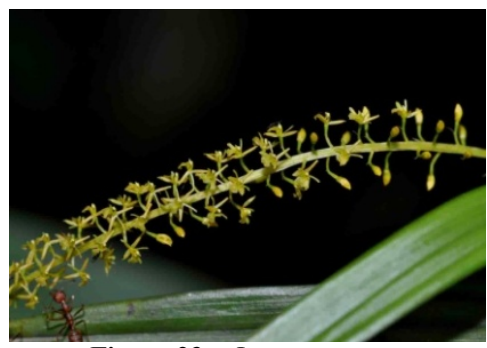

Figure 23. Liparis mannii

Liparis viridiflora (B1.) Lindl., Gen. Sp. Orchid. 31. 1830; Hook.f., Fl. Brit. India 5: 704. 1890.

Plant $12-23 \mathrm{~cm}$ tall. Pseudobulbs $1.8-3.5 \times 1.5-2.5 \mathrm{~cm}$, clustered, stem like, ovoid at base, long-cylindric above. Leaves $2,8-16 \times 2-3 \mathrm{~cm}$, obovate-oblong to oblanceolate, obtuse, acute or acuminate. Inflorescence cylindric, slender, densely many flowered, $6-11 \mathrm{~cm}$ long. Flowers small, greenish white or pale greenish yellow.

Habitat: Epiphyte in humid evergreen forest and mixed deciduous forest. Voucher specimen: Gogoi et al, 0073. Flowering: April- May. Local distribution: Joypur R. F, Assam. General distribution: India, Sri Lanka, Nepal, Bhutan, Burma, China, Thailand, Vietnam, Malaysia, Sumatra, Java, Borneo, New Guinea, Philippines, Fiji.

LUISIA Gaudichaud

About 40 species in tropical Asia, Malaysia, Australia and Japan; 16 species in India, 5 in Assam, 2 in Joypur.

Luisia trichorhiza (Hook. f.) Bl. Mus. Bot. 1: 63. 1849; Hook. f., Fl. Brit. India 6: 23. 1890; Chowdhery, Orch. Fl. Arunachal Prad. 495. 1998.

Plant $30-60 \mathrm{~cm}$ long. Stem stout, branched, covered by leaf sheaths. Leaves $7-16 \times 0.4-0.5 \mathrm{~cm}$, distichous, fleshy, terete, rugose, jointed. Inflorescence leaf-opposed, stout, 4 or 6-flowered, 6-8mm long. Flowers $0.8-1.1 \mathrm{~cm}$ across; sepals pale green with faint purple lines, lip dark purple, the base outlined with green lines, the apical ridges green, column purple.

Habitat: Epiphyte in humid evergreen forest. Voucher specimen: Gogoi et al, 0060. Flowering: March - May. Local distribution: Joypur R. F, Assam. General distribution: India, Nepal, Bangladesh, Bhutan, Burma, China, Thailand, Myanmar. (Figure 24)

Luisia zeylanica Lindl., Fol. Orchid. Luisia, 3. 1853;
Chowdhery, Orch. Fl. Arunachal Prad. 501. 1998.

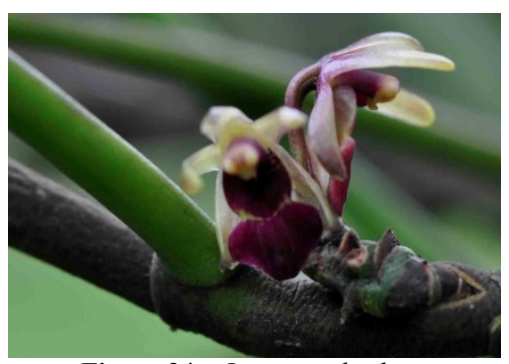

Figure 24. Luisia trichorhiza

Plant $23-37 \mathrm{~cm}$ tall. Stem stout, covered by leaf sheaths; internodes $1-1.5 \times 0.4-0.6 \mathrm{~cm}$. Leaves $6-15 \times 0.2-0.5 \mathrm{~cm}$, distichous, terete, subacute, sessile. Inflorescence extra-axillary, arising in centre of internode, on same side as the leaf, few flowered, $2-3 \mathrm{~mm}$ long, attenuate. Flowers greenish yellow, with purple base.

Habitat: Epiphyte in humid evergreen forest. Voucher specimen: Gogoi et al, 0496. Flowering: April- May. Local distribution: Joypur R. F, Assam. General distribution: India, Nepal, Bangladesh, Bhutan, Burma, China, Thailand, Myanmar, Sri Lanka, Cambodia, Vietnam, Borneo, Sumatra, Java, New Guinea, Philippines, Australia.

Malaxis Soland. ex Swartz

300 species distributed throughout the world, specially in S. E. Asia and adjoining Island; 19 species in India, 2 in Assam and 2 in Joypur.

Malaxis acuminata D. Don. Prodr. Fl. Nepal 29. 1852; Chowdhery, Orch. Fl. Arunachal Prad. 502. 1998.

Plant $15-20 \mathrm{~cm}$ tall. Stem glabrous, sheathed at base. Leaves 3 or $4,6-14 \times 2.8-7 \mathrm{~cm}$, thinly membranous, ovate-lanceolate, acute to acuminate, narrowed to shearhing base, petiolate, margins undulate. Inflorescence racemose, laxly many-flowered, $12-25 \mathrm{~cm}$ long, ribbed. Flowers 7-9mm long, uniformly purple.

Habitat: Terrestrial in dense humid evergreen forest on rocky terrain, also in the lowlands. Voucher specimen: Gogoi et al, 0499. Flowering: June - July. Local distribution: Joypur R. F, Assam. General distribution: India, Nepal, Bhutan, Burma, China, Thailand, Cambodia, Vietnam, Sumatra, Java, New Guinea, Philippines, Australia. (Figure 25)

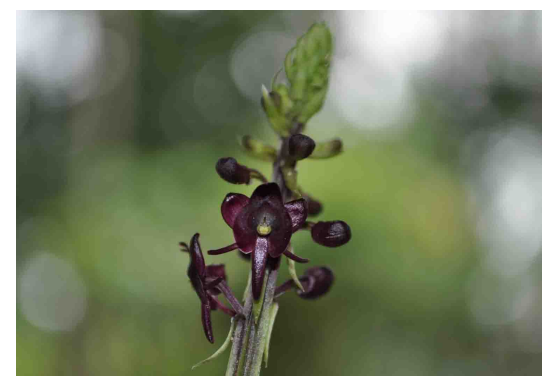

Figure 25. Malaxis acuminata

Malaxis latifolia J.E. Smith in Rees, Cyclop. 22, no. 3. 1819; Pradhan, Indian Orchid-II: 208. 1979; Chowdhery, Orch. Fl. Arunachal Prad. 504. 1998.

Plant $10-30 \mathrm{~cm}$ tall. Pseudobulbs $3.5-8 \times 1.5-2 \mathrm{~cm}$, 
enveloped in remnant of leaf sheaths. Leaves 3-6, lamina $6-18 \times 4-7.5 \mathrm{~cm}$, unequal, elliptic-lanceolate, acute or acuminate, channeled sheathing petiole, light green above and whitish green suffused with purple below, many-nerved. Inflorescence single, apical, $15-37 \mathrm{~cm}$ long, erect, striated. Flowers pale purple, $0.5 \mathrm{~cm}$ across.

Habitat: Terrestrial in open humid evergreen forest. Voucher specimen: Gogoi et al, 0200. Flowering: May June. Local distribution: Joypur R. F, Assam. General distribution: India, Nepal, Bhutan, Burma, China, Thailand, Myanmar, Sri Lanka, Cambodia, Vietnam, Borneo, Sumatra, Java, New Guinea, Philippines, Australia.

Micropera Lindley

3 species in India, 3 in Assam and 2 in Joypur.

Micropera mannii (Hook. f.) T. Tang \& F.T. Wang in Acta Phytotax. Sin. 1(1): 94. 1951; Pradhan, Indian Orchid-II: 497. 1979; Chowdhery, Orch. Fl. Arunachal Prad. 512. 1998.

Plant pendulous, branched, covered by leaf sheaths, $20-40 \mathrm{~cm}$ long. Leaves $8-12 \times 0.8-1.2 \mathrm{~cm}$, distichously, linear-oblong, keeled beneath, obtuse to subacute, minutely bifid at apex, sessile, jointed. Inflorescens straight, laxly 10-15 flowered, 8-10cm long. Flowers pale-pink, 0.6-0.7 cm across.

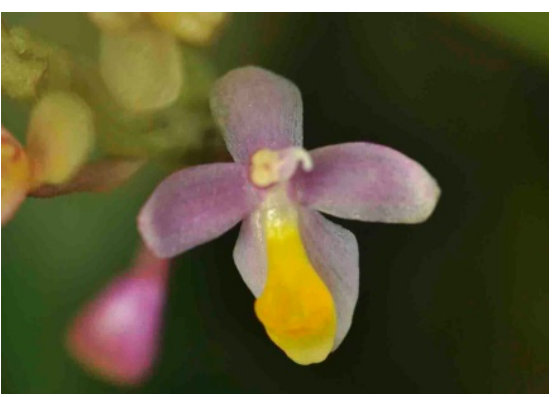

Figure 26. Micropera mannii

Habitat: Epiphyte on tree trunk in evergreen tropical forest. Voucher specimen: Gogoi et al, 0324. Flowering: June - July. Local distribution: Joypur R. F, Assam. General distribution: India (Assam), Bangladesh and Eastern Himalayas. (Figure 26)

Micropera rostrata (Roxb.) Balakr. in Jour. Bombay Nat. Hist. Soc. 67: 66. 1970; Mishra, Orch. India 307. 2007; Chowdhery, Orch. Fl. Arunachal Prad. 512. 1998.

Plant pendulous, branched, covered by leaf sheaths, $30-60 \mathrm{~cm}$ long. Leaves $7-10 \times 2-3 \mathrm{~cm}$, coriaceous, obtusely bifid at apex, sessile, jointed. Inflorescence laxly many flowered racemes, exceeding the leaves, $10-15 \mathrm{~cm}$ long, glabrous. Flowers pale-purple, $1.5-2 \mathrm{~cm}$ across.

Habitat: Epiphyte on tree trunk in evergreen tropical forest. Voucher specimen: Gogoi et al, 0312. Flowering: April May. Local distribution: Joypur R. F, Assam. General distribution: India (Assam), Bangladesh and Eastern Himalayas.

\section{Oberonia Lindley}

About 100 species mainly distributed to tropical Asia, a few are known from pacific Islands and Australia, a single species in Madagascar and tropical Africa; 50 species in
India, 13 in Assam, 1 in Joypur.

Oberonia mucornata (D. Don.) Ormerod \& Seidenfaden in Seidenfaden, Contr. Orchid Fl. Thailand 13: 20. 1997; Mishra, Orch. India 309. 2007.

Plant $21-34 \mathrm{~cm}$ tall. Stem very short. Leaves 4-6, $5-22 \times 1.7-2.5 \mathrm{~cm}$, broadly ensiform, acute to mucronate, overlapping at base, fleshy, the lower ones smaller. Inflorescence decurved, densely many-flowered, $15-24 \mathrm{~cm}$ long, slender, ridged. Flowers small, yellowish.

Habitat: Epiphyte on tree trunk in evergreen tropical forest. Voucher specimen: Gogoi et al, 0055. Flowering: September - October. Local distribution: Joypur R. F, Assam. General distribution: India, Bangladesh, Bhutan, Indonesia, Laos, China, Malaysia, Myanmar, Nepal, Philippines. (Figure 27)

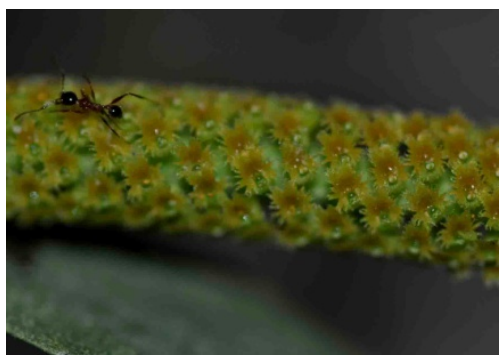

Figure 27. Oberonia mucornata

Papilionanthe Schlechter

About 11 species in India, S.E. Asia, and Malaya Archipelago; 4 species in India, 2 in Assam, 1 in Joypur.

Papilionanthe teres (Roxb.) Schltr. in Orchis 9: 78: 1915; Mishra, Orch. India 311. 2007; Chowdhery, Orch. Fl. Arunachal Prad. 570. 1998.

Plant $60-155 \mathrm{~cm}$ long, some times more, branched, terete, long, covered by leaf sheaths. Leaves $7-19 \times 0.6-0.8 \mathrm{~cm}$, suberect, curved, terete, linear, ridged, jointed. Inflorescence leaf-opposed, racemose, laxly 3 to 5 -flowered, $10-15 \mathrm{~cm}$ long, ridged, sheathed. Flowers $4.8-7.5 \mathrm{~cm}$ across; sepals and petals whitish pink, lip darker pink with a yellow base veined with pink, spur with a yellow mouth.

Habitat: Epiphyte in mixed deciduous forest, also on solitary roadside trees. Voucher specimen: Gogoi et al, 0059. Flowering: April - May. Local distribution: Joypur R. F, Assam. General distribution: N.E. India, Nepal, Bhutan, Burma, China, Thailand. (Figure 28)

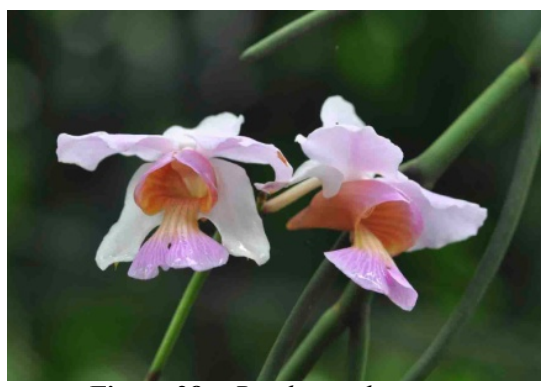

Figure 28. Papilionanthe teres

PHAIUS Loureiro

7 species in India, 4 in Assam, 2 in Dibrugarh district.

Phaius mishmensis Reichb. f. in Bonplandia 5: 43. 1857; Hook. f., Fl. Brit. India 5: 817. 1890; Mishra, Orch. India 313. 
2007; Chowdhery, Orch. Fl. Arunachal Prad. 583. 1998.

Plant $40-123 \mathrm{~cm}$ tall. Pseudobulbs $3.5-6.7 \times 1.3-2 \mathrm{~cm}$, narrowly fusiform-cylindric, fleshy. Leaves 6-8, 14-34×5.5-11 cm, elliptic-lanceolate to oblong-ovate, acuminate to acute, sheathing at base. Inflorescence 1 or 2 , $15-20 \mathrm{~cm}$ long, axillary, laxly few-flowered, weakly zigzag, sheathed. Flowers $4-5 \mathrm{~cm}$ across, white, greenish yellow or pinkish purple.

Habitat: Terrestrial, on the bank of a small stream in dense humid evergreen forest. Voucher specimen: Gogoi et al, 0484. Flowering: November- January. Local distribution: Joypur R. F, Assam. General distribution: N.E. India, Bhutan, Burma, China, Ryukyu Arch., Vietnam, Thailand. (Figure 29)

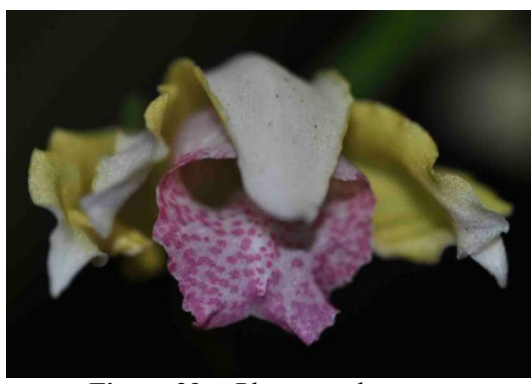

Figure 29. Phaius mishmensis

Phaius tancarervilliae (Banks ex L'Herit.) B1., Mus. Bot. Lugd.- Bat. 2: 177. 1856; Mishra, Orch. India 313. 2007; Chowdhery, Orch. Fl. Arunachal Prad. 583. 1998.

Plant $54-100 \mathrm{~cm}$ tall. Pseudobulbs $3.5-7 \mathrm{~cm}$ long, conical to ovoid, green, sheathed by leaf bases. Leaves 4-5, $30-75 \times 9-18 \mathrm{~cm}$, elliptic-lanceolate, acuminate, thin-textured; petiole $11-19 \mathrm{~cm}$ long. Inflorescence $23-95 \mathrm{~cm}$ tall, lateral, laxly 6 to 20 -flowered. Flowers $7-12 \mathrm{~cm}$ across; sepals and petals whitish outside, reddish brown or brown inside; lip white toward base, pink or red-pink toward entrance with white stripes on inside.

Habitat: Terrestrial, shaded and damp places in forests. Voucher specimen: Gogoi et al, 0512. Flowering: JanuaryMarch. Local distribution: Joypur R. F, Assam. General distribution: India, Sri Lanka, Bhutan, Burma, China, Japan, Thailand, Cambodia, Vietnam, Peninsular Malaysia, Sumatra, Java, Borneo, New Guinea, Philippines, Australia, Pacific Is.

\section{Phalaenopsis Blume}

About 46 species in India, S. E. Asia, Indonesia, Philippines and N. Australia; 7 species in India, 3 in Assam, 2 in Joypur.

Phalaenopsis mannii Reichb. f. in Gard. Chron. 902. 1871; Hook. f., Fl. Brit. India, 6: 30, 1890; Chowdhery, Orch. Fl. Arunachal Prad., 587, 1998.

Plant pendulous, stem short, enclosed by overlapping leaf sheaths. Leaves 4-5, 10-30×4-7.5cm, coriaceous, oblong-lanceolate to narrowly oblong, acute or obtuse, sessile, jointed. Inflorescence 1-4, 12-31 cm long, slender, pendent, racemose or paniculate, laxly 6 to 14-flowered. Flowers 3-4 cm across; sepals and petals yellow with dark brown spots and bars; lip mid-lobe white; column yellow.
Habitat: Epiphytic on tree trunks in evergreen forests. Voucher specimen: Gogoi et al, 0344. Flowering: March May. Local distribution: Joypur R. F, Assam. General distribution: N.E. India, Bhutan, China, Myanmar, Nepal, Vietnam.

Phalaenopsis parishii Reichb. f. in Gard. Chron. 410. 1865; Hook.f. Fl. Brit. India 6: 31. 1890; Chowdhery, Orch. Fl. Arunachal Prad. 587. 1998.

Plant epiphyte; roots copious, flattened; stems clustered, abbreviated, branching basally. Leaves $2-4,5-8 \times 3.5-4 \mathrm{~cm}$, subbasal, broadly elliptic, obliquely bilobed. Inflorescences erect racemes, 5-10 cm, 4-8 flowered. Flowers white with two chestnut bands on lip, $2 \mathrm{~cm}$ across.

Habitat: Epiphytic on tree trunks in open forests. Voucher specimen: Gogoi et al, 0258. Flowering: March - April. Local distribution: Joypur R. F, Assam. General distribution: India, China, Bhutan, Myanmar, Vietnam. (Figure 30)

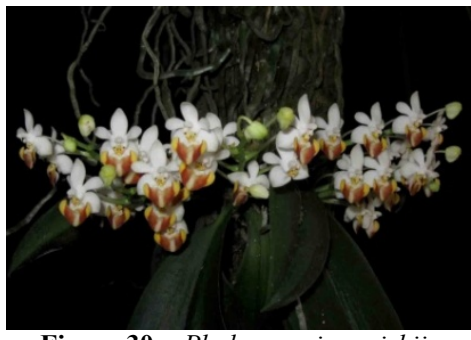

Figure 30. Phalaenopsis parishii

Pholidota Lindl. ex. Hook. f.

About 30 species in India, China, Malaysia, Indonesia, New Guinea, Pacific Islands and Australia; 10 species in India, 7 in Assam, 2 in Joypur.

Pholidota articulata Lindl. Gen. Sp. Orchid. 38,1833; Hook. f., Fl. Brit. India, 5: 844, 1890.

Plant $22-43 \mathrm{~cm}$ long. Pseudobulbs $4-9 \mathrm{~cm}$ long, slender to swollen; internodes 1.9-5mm long. Leaves 2, $6.5-11 \times 2-3.7 \mathrm{~cm}$, ovate to linear-lanceolate, acute to acuminate. Inflorescence synanthous, many flowered, $4-7 \mathrm{~cm}$ long, straight to zigzag. Flowers greenish white or white and slightly tinged with reddish, $5 \mathrm{~mm}$ across.

Habitat: Epiphyte in dense humid evergreen forest close to a waterfall. Voucher specimen: Gogoi et al, 0435. Flowering: July - October. Local distribution: Joypur R. F, Assam. General distribution: India, Nepal, Bhutan, Burma, China (Yunnan), Thailand, Cambodia, Vietnam, Peninsular Malaysia, Sumatra, Java, Borneo, Sulawesi, Philippines.

Pholidota imbricata (Roxb.) Lindl. in Hook., Fl. Exot. 2: t. 138. 1825; Hook. f., Fl. Brit. India 5: 845. 1890; Chowdhery, Orch. Fl. Arunachal Prad. 589. 1998.

Plant $16-41 \mathrm{~cm}$ tall. Pseudobulbs 5-11x1.8-2cm, ovoidconical, sheathed at base. Leaf solitary, $19-38 \times 4.7-8.5 \mathrm{~cm}$, arises from pseudobulb apex, oblong, obovate-oblong to linear-lanceolate, acute to acuminmate, coriaceous. Inflorescence synanthous, densely many-flowered, $17-20 \mathrm{~cm}$ long. Flowers white, $5 \mathrm{~mm}$ across.

Habitat: Epiphyte on tree humid evergreen forest. Voucher specimen: Gogoi et al, 0106. Flowering: June August. Local distribution: Joypur R. F, Assam. General 
distribution: India, Nepal, Bhutan, Burma, China, Thailand, Cambodia, Vietnam, Peninsular Malaysia, Sumatra, Java, Borneo, New Guinea, Philippines, Australia. (Figure 31)

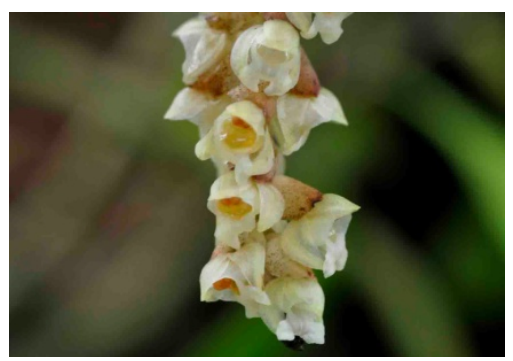

Figure 31. Pholidota imbricata

\section{Podochilus Blume}

4 species in India, 2 in Assam, 2 in Joypur.

Podochilus cultratus Lindl., Gen Sp. Orchid. 234. 1833; Hook. f., Fl. Brit. India 6: 80. 1890.

Plant $7-15 \mathrm{~cm}$ long, glabrous, completely covered by leaves. Leaves many, $0.9-1.2 \times 0.6-0.8 \mathrm{~cm}$, equitant, lanceola te, falcate, acute, many-veined. Inflorescence slender, 2 to 4-flowered, 1.1-1.2 $\mathrm{cm}$ long. Flowers white, $5 \mathrm{~mm}$ long.

Habitat: Epiphyte on tree trunk in wet evergreen tropical forest. Voucher specimen: Gogoi et al, 0497. Flowering: April- May. Local distribution: Joypur R. F, Assam. General distribution: N.E. India, Nepal, Myanmar, Thailand and Vietnam.

Podochilus khasianus Hook. f., Fl. Brit. India 6: 81. 1890; Chowdhery, Orch. Fl. Arunachal Prad. 616. 1998.

Plant $9-14 \mathrm{~cm}$ long slender, tufted, suberect, covered by sheathing leaf bases. Leaves many, $0.9-1.3 \times 0.0 .2-0.3 \mathrm{~cm}$, linear to linear-oblong, acute, coriaceous, jointed, sometimes twisted, sheathing at base. Inflorescence 3-5 flowered, $0.4-0.6 \mathrm{~cm}$ long, glabrous, weakly zigzag. Flowers white, very small.

Habitat: Epiphyte on tree trunk in wet evergreen tropical forest. Voucher specimen: Gogoi et al, 0400. Flowering: June - August. Local distribution: Joypur R. F, Assam. General distribution: N.E. India, Bhutan, China, Vietnam. (Figure 32)

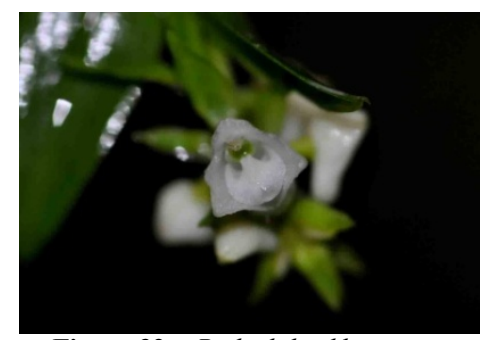

Figure 32. Podochilus khasianus

Promatocalpa Breda, Kuhl \& Hasselt

8 species in India, 1 in Assam and Dibrugarh district.

Promatocalpa undulatum (Reichb. f.) J.J. Smith in Nat. Tijdschr. Ned. Ind. 72: 107. 1912; Chowdhery, Orch. Fl. Arunachal Prad. 622. 1998; Mishra, Orch. India 315. 2007.

Plant upto $28 \mathrm{~cm}$ long covered by leaf sheaths. Leaves $7-15 \times 1.5-2 \mathrm{~cm}$, narrowly oblong to oblong-elliptic, apex obscurely 2-lobed, acuminate to obliquely obtuse.
Inflorescence racemose, many flowered, $2-2.5 \mathrm{~cm}$ long, narrow, glabrous, sheathed. Flowers $8 \mathrm{~mm}$ across, yellow with purple blotched; lip with purple blotched.

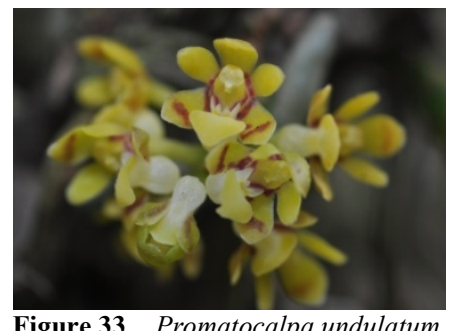

Habitat: Epiphyte in the wet evergreen tropical forest. Voucher specimen: Gogoi et al, 0498. Flowering: MarchMay. Local distribution: Joypur R. F, Assam. General distribution: N.E. India, Bangladesh and China. (Figure 33)

Pteroceras Hasselt ex Hassk.

5 species in India, 1in Assam and Joypur.

Pteroceras suaveolens (Roxb.) Holtt. in Kew Bull. 14: 271. 1960; Chowdhery, Orch. Fl. Arunachal Prad. 624. 1998.

Plant pendulous, $15-24 \mathrm{~cm}$ long, unbranched. Leaves 4-12, $5-15 \times 1.5-3 \mathrm{~cm}$, linear-oblong to lanceolate, oblique, bilobed, sessile, jointed. Inflorescence 1 or many, 4-12-flowered, $4.5-7.5 \mathrm{~cm}$ long, terete, glabrous, dilated, nodes winged. Flower yellow with brownish spots, about $1.5 \mathrm{~cm}$ across; lip tip dark purple.

Habitat: Epiphyte in the wet evergreen tropical forest. Voucher specimen: Gogoi et al, 0489. Flowering: June - July. Local distribution: Joypur R. F, Assam. General distribution: India (NE), Nepal, Burma, Thailand, Cambodia, Vietnam, Peninsular Malaysia, Sumatra, Java, Borneo, Philippines. (Figure 34)

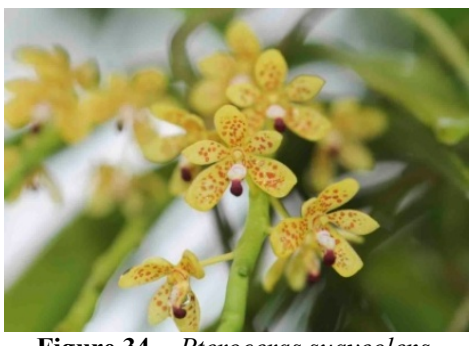

Figure 34. Pteroceras suaveolens

\section{Rhynchostylis Blume}

About 4 species distributed in India, Srilanka, Myanmar, S. E. Asia, Malaysia, Philippines and Indonesia; 2 in India and Assam, 1 in Joypur.

Rhynchostylis retusa (Lind1.) B1., Bijdr. 286, t. 49. 1825; Hook. f., Fl. Brit. India 6: 32. 1890; Chowdhery, Orch. Fl. Arunachal Prad. 626. 1998.

Plant $8-31 \mathrm{~cm}$ long, some times more, stout, covered by leaf sheaths. Leaves distichous, $12-40 \times 2-3.8 \mathrm{~cm}$, spreading to arching, lorate, apex obliquely 2-lobed, coriaceous, deeply channeled, sessile, jointed. Inflorescence $20-40 \mathrm{~cm}$ long, densely many-flowered. Flowers $1.7-2.3 \mathrm{~cm}$ across; sepals and petals white and spotted with pink or pale purple; lip purplish pink, apex white.

Habitat: Epiphyte in the wet evergreen tropical forest. 
Voucher specimen: Gogoi et al, 0049. Flowering: May June. Local distribution: Joypur R. F, Assam. General distribution: India, Sri Lanka, Nepal, Burma, China, Thailand, Cambodia, Vietnam, Peninsular Malaysia, Sumatra, Java, Philippines. (Figure 35)

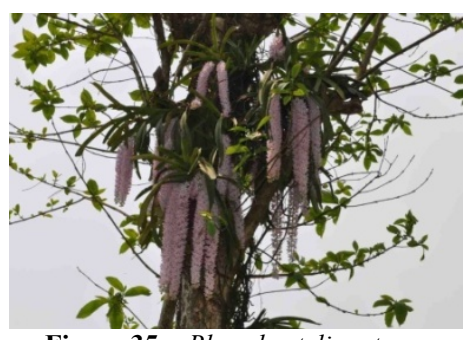

Figure 35. Rhynchostylis retusa

Robiquetia Gaudichaud

About 25 species in Indonesia, Malaysia, Philippines, Papua New Guinea, Australia and Pacific Island; 4 species in India, 2 in Assam, 1 in Joypur.

Robiquetia spathulata (B1.) J.J. Smith in Nat. Tijdschr. Ned. Ind. 72: 115. 1912; Chowdhery, Orch. Fl. Arunachal Prad. 630. 1998; Mishra, Orch. India 315. 2007.

Plant $60-90 \mathrm{~cm}$ long, pendulous, covered in leaf sheaths, $0.8-1.2 \mathrm{~cm}$ thick. Leaves $10-18 \times 3.6-4.5 \mathrm{~cm}$, broadly oblongelliptic, apex unequally and broadly bilobed, narrowed to sessile, jointed base. Inflorescence racemose, densely many-flowered, $10-15 \mathrm{~cm}$ long. Flowers yellow with purplish brown spots and stripes.

Habitat: Epiphyte humid evergreen forest. Voucher specimen: Gogoi et al, 0450. Flowering: May - July. Local distribution: Joypur R. F, Assam. General distribution: N.E. India, Burma, China, Thailand, Cambodia, Vietnam, Peninsular Malaysia, Sumatra, Java, Borneo, Philippines.

\section{Taeniophyllum Blume}

7 species in India, 2 in Assam and 1 in Dibrugarh district.

Taeniophyllum crepidiforme (King \& Pantl.) King \& Pantl. in Ann. Roy. Bot. Gard. Calcutta 8: 245, t. 325. 1898; Chowdhery, Orch. Fl. Arunachal Prad. 656. 1998; Mishra, Orch. India 317. 2007.

Plant small, stemless, leafless, epiphytic herb devoid of pseudobulbs with comparatively thick, flat greenish roots; Inflorescence $7-9 \mathrm{~mm}$ long, filiform. Flowers minute, greenish white with purple tinge.

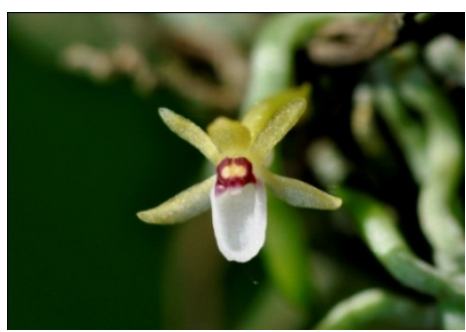

Figure 36. Taeniophyllum crepidiforme

Habitat: Epiphyte in humid evergreen forest. Voucher specimen: Gogoi et al, 0506. Flowering: August- September. Local distribution: Joypur R. F, Assam. General distribution: Eastern Himalayas, Sikkim and Assam. (Figure 36)

Tainia Blume
About 50 species in India China, S. E. Asia; 9 species in India, 4 in Assam, 3 in Joypur.

Tainia latifolia (Lindl.) Benth. ex. Hook. f., Fl. Brit. India 5: 820. 1890; Chowdhery, Orch. Fl. Arunachal Prad. 657. 1998.

Plant $80-140 \mathrm{~cm}$ tall. Pseudobulbs $7-1 \times 0.5-1.3 \mathrm{~cm}$, cylindric-ovoid, swollen towards base. Leaf $14-38 \times 5-9 \mathrm{~cm}$, solitary, petiolate, elliptic to ovate-obovate, acute to acuminate, margins entire, many-veined. Inflorescence arising from above base of pseudobulb, many flowered, 40-50 cm long. Flowers $2 \mathrm{~cm}$ across, dark brown, lip yellow.

Habitat: Terrestrial in dense humid evergreen forest. Voucher specimen: Gogoi et al, 0350. Flowering: March May. Local distribution: Joypur R. F, Assam. General distribution: N.E. India, Bangladesh, China, Bhutan, Burma, Thailand, Vietnam, Myanmar.

Tainia minor Hook. f. Fl. Brit. India 5: 821. 1890; Chowdhery, Orch. Fl. Arunachal Prad. 661. 1998.

Plant $23-36 \mathrm{~cm}$ tall. Pseudobulbs $3.5-6.8 \times 0.7-1.1 \mathrm{~cm}$, cylindric, expanded at base, scale-bracts at apex persistent. Leaf solitary, $13-25 \times 3.5-5.7 \mathrm{~cm}$, elliptic, acuminate, manyveined, petiolate. Inflorescence terminal, many flowered, $11-23 \mathrm{~cm}$ long, sheathed. Flowers $1.5 \mathrm{~cm}$ across, purplish brown with deep purple spots; lip white, tinged with purplish brown.

Habitat: Terrestrial in dense humid evergreen forest. Voucher specimen: Gogoi et al, 0488. Flowering: JuneAugust. Local distribution: Joypur R. F, Assam. General distribution: N.E. India, China, Myanmar.

Tainia waryana (Hook. f.) J.J. Smith in Bull. Jard. Bot. Buitenz. 2, 8: 6. 1912; Pradhan, Indian Orchid-II: 242. 1979; Chowdhery, Orch. Fl. Arunachal Prad. 661. 1998.

Plant $10-15 \mathrm{~cm}$ long including leaves. Pseudobulbs 8-10c $\mathrm{m}$ long, narrowly fusiform, one leaved, with membranous sheaths. Leaves solitary, $10-15 \times 7-9 \mathrm{~cm}$, ovate-elliptic, cordate at base, 7-9 nerved, petioles stout, terete.Flowers greenish flushed with red, $3 \mathrm{~cm}$ across; lip white.

Habitat: Terrestrial in dense humid evergreen forest. Voucher specimen: Gogoi et al, 0481. Flowering: June - July. Local distribution: Joypur R. F, Assam. General distribution: N.E. India, Thailand, Malaysia to Sumatra. (Figure 37)

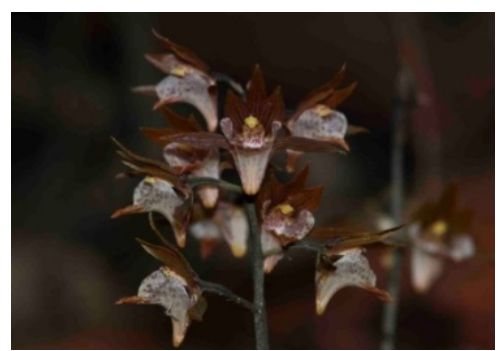

Figure 37. Tainia waryana

Thelasis Blume

4 species in India, 2 in Assam and Joypur.

Thelasis longifolia Hook. f., Fl. Brit. India 6: 87. 1890; Chowdhery, Orch. Fl. Arunachal Prad. 662. 1998.

Plant $16-23 \mathrm{~cm}$ tall. Pseudobulbs $1.3-2.5 \times 1.2-2.7 \mathrm{~cm}$, conical. Leaf solitary, $9-18 \times 1.3-2.2 \mathrm{~cm}$, arising from apex of 
the pseudobulb, narrowly oblong to oblanceolate, minutely bifid at the apex or rounded, sessile. Inflorescence arising from newly developing pseudobulb, many flowered, longer than leaves, sheathed at base, $14-19 \mathrm{~cm}$ long, glabrous, sheathed. Flowers green, not opening widely, small.

Habitat: Epiphytic in evergreen forest. Voucher specimen: Gogoi et al, 0355. Flowering: June- August. Local distribution: Joypur R. F, Assam. General distribution: N.E. India, Himalayas.

Thelasis pygmaea (Griff.) Lindl. in Jour. Proc. Linn. Soc. 3: 63. 1859; Hook. f., Fl. Brit. India 6: 86. 1890; Chowdhery, Orch. Fl. Arunachal Prad. 662. 1998.

Plants $6-10 \mathrm{~cm}$ tall. Pseudobulbs $0.8-1.4 \times 0.9-1 \mathrm{~cm}$, ovoid -globose. Leaves 1 or $2,3-7.5 \times 1.2-1.4 \mathrm{~cm}$, linear-oblong, sessile, fleshy, tip slightly biforked. Inflorescence lateral, $5-9 \mathrm{~cm}$ long, arising from base of pseudobulbs, densely many-flowered, 4-6cm long, sheathed. Flowers yellowish green, not opening widely, small.

Habitat: Epiphytic in mixed deciduous and evergreen forest. Voucher specimen: Gogoi et al, 0487. Flowering: July- September. Local distribution: Joypur R. F, Assam. General distribution: India, Nepal, Burma, China, Thailand, Vietnam, Peninsular Malaysia, Sumatra, Java, Borneo, New Guinea, Philippines.

Thrixspermum Loureiro

About 100 species distributed widely from the Himalayas to Philippines and the tropical Islands of S.W. Pacific; 9 species in India, 3 in Assam, 3 in Joypur.

Thrixspermum acuminatissimum (B1.) Reich. f. Xen. Orchid. 2: 121. 1867; Scidenfaden et al, Orchid. Thailand iv-I: 518.1962.

Plant monopodial. stem very short, $1-2 \mathrm{~cm}$ long. Leaves $3.5-5 \times 1-1.5 \mathrm{~cm}$, distichous, subsessile, bilobed at apex, articulated to sheathing leaf base, coriaccous, oblong; peduncles $10-12 \mathrm{~cm}$ long, arising from one point on the stem, curved, naked. Inflorescence raceme, 5-6.5 cm long, bracts ovate, incurved coriaccous, subacute; pedicel and ovary $0.7 \mathrm{~cm}$ long, slender. Flowers yellow; lip white with yellow and reddish-brown spots.

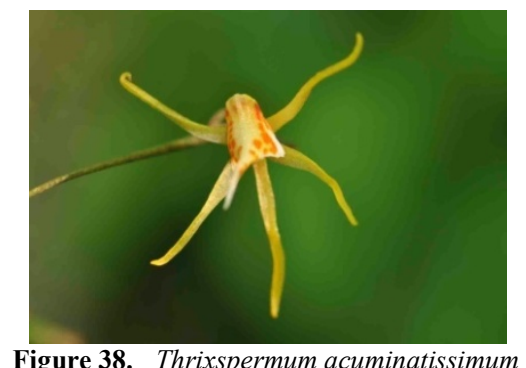

Habitat: Growing on lateral branches of lower canopy of host tree, cool and shady place. Voucher specimen: Gogoi et al, 0510. Flowering: July- December. Local distribution: Joypur R. F, Assam. General distribution: India (Assam), Thailand, Cambodia, S. Vietnam, Malacca, Java, Sumatra, Kalimantan, Philippines. (Figure 38)

Thrixspermum centipeda Lour., Fl. Cochinch. 520. 1790; Chowdhery, Orch. Fl. Arunachal Prad. 665. 1998;
Chowdhery \& Pal, Orch. Arunachal Prad. 140. 1997.

Plant erect, covered by persistent leaf sheaths; internodes $1.5-2 \mathrm{~cm}$ long. Leaves $7-9,8-11 \times 2-2.6 \mathrm{~cm}$, coriaceous, linear-oblong, apex minutely bifid, jointed. Inflorescence leaf-opposed, 5-10cm long, 1 or 2-sheathed, glabrous; rachis $4-12 \mathrm{~cm}$ long, laterally flattened. Flowers white or creamy yellow, turning yellow later.

Habitat: Epiphyte in mixed deciduous forest, in humid evergreen forest. Voucher specimen: Gogoi et al, 0430. Flowering: May - August. Local distribution: Joypur R. F, Assam. General distribution: N.E. India, Burma, China, Thailand, Cambodia, Vietnam, Peninsular Malaysia, Sumatra, Java, Borneo, Philippines.

Thrixspermum pygmaeum (King \& Pantl.) Holtt. in Kew Bull. 14: 275. 1960; Chowdhery, Orch. Fl. Arunachal Prad. 666. 1998; Mishra, Orch. India 318. 2007.

Plant $2-4 \mathrm{~cm}$ tall, covered by leaf sheaths. Leaves $2-5$, 2-7 $\times 0.4-0.9 \mathrm{~cm}$, linear-oblong, acuminate, jointed.Infloresc ence distantly 1-2 flowered, $1.5-2 \mathrm{~cm}$ long, slender, minutely sheathed. Flowers about $1 \mathrm{~cm}$ across, white.

Habitat: Epiphyte in mixed deciduous forest, in humid evergreen forest. Voucher specimen: Gogoi et al, 0714. Flowering: May - August. Local distribution: Joypur R. F, Assam. General distribution: N.E. India, Burma, China, Thailand, Cambodia, Vietnam, Peninsular Malaysia, Sumatra, Java, Borneo, Philippines.

Trichotosia Blume

50 species widely distributed from Himalayas to the S.W. Pacific Island; 3 species in India, 2 in Assam and 1 in Joypur.

Trichotosia velutina (Lodd. ex Lindl.) Kranz. in Engler \& Prantl, Pflanzenr. 50: 140. 1911; Chowdhery, Orch. Fl. Arunachal Prad. 672. 1998; Mishra, Orch. India 318. 2007.

Plant 40-60 cm long, pendulous, coverd with persistent sheaths and tubular leaf bases, reddish brown tomentose. Leaves, many, alternate, well spread over the stem, 5-8 cm long, oblong-lanceolate, thickly coriaceous. Inflorescence leaf opposed, short, 3-6 flowered. Flowers pale- white, $1 \mathrm{~cm}$ across.

Habitat: Epiphyte on tree trunks in humid forest. Voucher specimen: Gogoi et al, 0391. Flowering: August - September. Local distribution: Joypur R. F, Assam. General distribution: N.E. India, Burma, Thailand, Malaysia, Sumatra, Java, New Guinea and Borneo.

Tropidia Lindley

3 species in India, 2 in Assam and 1 in Joypur.

Tropidia curculigioides Lindl., Gen. Sp. Orchid. 497. 1840; Chowdhery, Orch. Fl. Arunachal Prad. 676. 1998.

Plant $33-65 \mathrm{~cm}$ tall. Stem slender. Leaves $9-22 \times 2-3 \mathrm{~cm}$, oblong-elliptic, tapering, acute to acuminate, sessile with sheathing base, plicate. Inflorescence short, leaf-opposed, subglobose, densely flowered. Flowers greenish white, 1.5 cm long.

Habitat: Terrestrial in dense humid evergreen forest and evergreen montane forest. Voucher specimen: Gogoi et al, 0479. Flowering: September- November. Local distribution: Joypur R. F, Assam. General distribution: India, Burma, China, Taiwan, Thailand, Cambodia, Vietnam, Malaysia, 
Java, Borneo, New Guinea, Australia.

Tylostylis Blume

1 species in India, Assam and Joypur.

Tylostylis discolour (Lindl.) Hook. f. in Ann. Roy. Bot. Gard. Calcutta 5: 22. 1895; Pradhan, Indian Orchid-II: 344. 1979; Chowdhery, Orch. Fl. Arunachal Prad. 677. 1998.

Plant $25-33 \mathrm{~cm}$ tall; rhizome thick, woody, sheathed. Pseudobulbs $10-21 \times 2.5-3.6 \mathrm{~cm}$, thick, cylindric, furrowed. Leaves 3-5, 10-18×2.7-4cm, arising from pseudobulbs apex, narrowly oblong to elliptic, emarginated at apex, sessile. Inflorescence lateral, few-flowered, $3-8 \mathrm{~cm}$ long, erect to arching, sheathed, pubescent. Flowers few, $1.3-1.6 \mathrm{~cm}$ across, tomentose externally, yellow-ochre, lip with a dark purplish patch near base.

Habitat: Epiphytic on trees in mixed forests. Voucher specimen: Gogoi et al, 0201. Flowering: January- March. Local distribution: Joypur R. F, Assam. General distribution: India, China, Indonesia, Laos, Malaysia, Myanmar, Thailand, Vietnam.

\section{Zeuxine}

There are about 50 species (King and Pantling, 1898; Garay and Sweet, 1974; Seidenfaden, 1978; Bose and Bhattacharjee, 1980; Hashimoto and Kanda, 1981; Davis and Steiner, 1982; Kanda, 1984; Deva and Naithani, 1986; Comber, 2001) to 90 species (Ormerod, 2005) of Zeuxine in the tropics and subtropics of the old World of which 19 species has been estimated from India (Misra, 2007). 9 species were recognized in Assam (Chowdhury, 2005) and 5 in Joypur.

Zeuxine clandestina Bl., Coll. Orchid. 70. 1858; Barua, Orch. fl. Kamrup Dist. 56. 2001; Mishra, Orch. India 320. 2007.

Plant $10-15 \mathrm{~cm}$ tall, stems and peduncles glabrous. Leaves $2-4,3-4 \times 2 \mathrm{~cm}$, linear, glabrous, 3 nerved, sheathed, scarious. Inflorescence spikes, 8-10 flowered. Flowers greenishwhite, small.

Habitat: Growing in dense forest, cool and shady place. Voucher specimen: Gogoi et al, 0508. Flowering: JunuaryMarch. Local distribution: Joypur R. F, Assam. General distribution: India, Thailand, Malaya and Java.

Zeuxine glandulosa King \& Pantling in Ann. Roy. Bot. Gard. (Calcutta) 8: 288. t. 384. 1898; Pradhan, Indian Orchid-I: 118. 1969; Mishra, Orch. India 320. 2007.

Plants 5-20 cm high. Leaves oblong - lanceolate, acute, shortly petiolate, $2.5-5 \times 0.6-1 \mathrm{~cm}$, blackish- purple; petiole expanded into a wide hyaline sheath. Inflorescence laxly few flowered. Flowers $0.5 \mathrm{~cm}$ long olivaceous green, column and central contracted part of lip white.

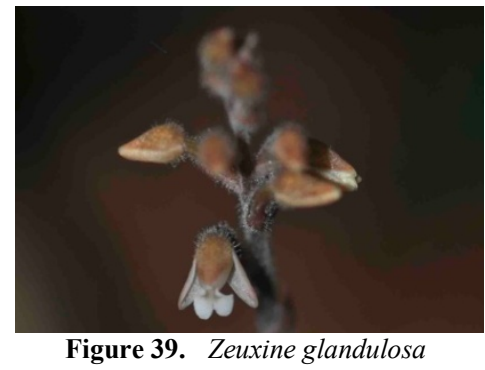

Habitat: Growing in dense forest, cool and shady place. Voucher specimen: Gogoi et al, 0511. Flowering: MarchApril. Local distribution: Joypur R. F, Assam. General distribution: Bhutan, Sikkim Himalaya, North Bengal and Assam. (Figure 39)

Zeuxine goodyeroides Lindl. Gen. Sp. Orchid. 486. 1840; Hook. f., Fl. Brit. India 6: 107. 1890.

Plant $15-27 \mathrm{~cm}$ tall, sheaths $0.7-1.1 \mathrm{~cm}$, clasping. Leaves $3-5,2.6-4 \times 1.2-2 \mathrm{~cm}$, oblong-ovate, acute, shortly petiolate. Inflorescence laxly many flowered, $4-10 \mathrm{~cm}$ long, ridged, sparsely pubescent, with 1 or 2 linear-lanceolate sheaths. Flowers resupinate, white, small.

Habitat: Growing in dense forest, cool and shady place. Voucher specimen: Gogoi et al, 0483. Flowering: JanuaryFebruary. Local distribution: Joypur R. F, Assam. General distribution: Himalayas, Nepal, Myanmar and Vietnam. Present ecological status: Common.

Zeuxine longilabris (Lindl.) Trim. in Joour. Ceylon Br. Roy Asiat. Soc. 9: 90. I885; Hook. f., Fl. Brit. India 6: 107. 1890; Chowdhery, Orch. Fl. Arunachal Prad. 695. 1998.

Plant $15-20 \mathrm{~cm}$ tall. Leaves $2-3 \times 1-2 \mathrm{~cm}$, ovate to ovate-oblong, acute, 5-7 nerved, drooping, copper-brown white, shortly petioled. Inflorescence terminal, fewflowered, 2-6cm long, glandular-pubescent racemes on elongate, slender, purple scapes. Flowers greenish-white, small.

Habitat: Terrestrial, growing in grassland. Voucher specimen: Gogoi et al, 0509. Flowering: March - May.

Local distribution: Joypur R. F, Assam. General distribution: India, Nepal, Sri Lanka, Bangladesh, Thailand, Myanmar and Cambodia.

Zeuxine nervosa (Wall. ex Lindl.) Benth. ex Trimen, J. Ceylon Branch Roy. Asiat. Soc. 9: 90 (1885).

Plants 20-35 cm tall, slender, 3-6-leaved. Leaves clustered at stem apex, not usually withering at anthesis, ovate to ovate-elliptic, $4-6 \times 1.5-2.5 \mathrm{~cm}$, apex acute. Inflorescence $18-28 \mathrm{~cm}$, with 2 or 3 widely spaced sterile bracts, sparsely villous, many flowered. Flowers resupinate, white, small.

Habitat: Damp places in forests. Voucher specimen: Gogoi et al, 0713. Flowering: January-March. Local distribution: Joypur R. F, Assam. General distribution: India, Sri Lanka, Eastern Himalayas, Nepal, Thailand, Taiwan, China, Bangladesh, Vietnam, Ryukyu Island, New Guinea and Philippines.

\section{Results and Discussion}

During recent field survey, 107 Orchid species with 47 genera have been recorded from different part of the Joypur reserve forest of Dibrugarh district of Assam. Of them, 82 are epiphytic and 23 are terrestrial and 2 species like Didymoplexis pallens and Epipogium roseum are saprophytic in habitat. Out of them 30 species is very common in this region but others are rare in occurrence. It is observed that whole Orchidaceae family facing high risk of 
threat in habitat in comparison with other plant species in the study areas. Rapid destruction of natural habitat by many means like deforestation, extension of agricultural lands, urbanization, developmental schemes, atmospheric pollution, pesticidal and weedicidal pollution and many more anthropogenic activities in the study areas are the main reasons. Therefore, protection and conservation of natural habitat are the only prominent way to save our precious natural wealth like Orchid species of the regions.

\section{ACKNOWLEDGEMENTS}

The authors are grateful to Dr. Gojen Chandra Sarma, Dept. of Botany, Guwahati University, Assam, Dr. Kashmira Kakoti, Dr. A. Cristy Williums, Deputy CommissionerDibrugarh and Divisional Forest Officer- Dibrugarh, Assam for constant supervision and valuable suggestions during the course of present studies.

\section{REFERENCES}

[1] Banerji TL, 1978, Orchids of Nepal. Bishen Singh Mahendra Pal Singh, Dehra Dun, India.

[2] Barua IC, 2001, Orchid Flora of KamrupDistrict. Bishen Singh Mahendra Pal Singh, Dehra Dun India.

[3] Bhagabati AK., MC Kalita and S Baruah, 2006, Biodiversity of Assam. Assam Science Society, Guwahati, Assam, India.

[4] Bruhl Paul, 1926, A Guide to the Orchid of Sikkim. Thacker \& Spink \& Co., Simla, India.

[5] Chowdhery HJ, 1998, Orchid Flora of Arunachal Pradesh. Bishen Singh Mahendra Pal Singh, Dehra Dun, India.

[6] Chowdhery S, 2005, Assam's Flora. Assam Science Technology and Environment Council, Guwahati, Assam, India.

[7] Deorani SC and HB, Naithani, 1995, Orchids of Nagaland. Oriental Enterprises, Dehra dun, India,

[8] Deva Som and HB, Nathani, 1968, The Orchid Flora of North-West Himalaya. Bishen Singh Mahendra Pal Singh, Dehra Dun, India.

[9] Gamble JS, 1928, Flora of presidency of Madras. Vol-III. Adlard and Sons Ltd., London.

[10] Gogoi K, 2005, The Genus Dendrobium in Dibru-Saikhowa National Park and Biosphere Reserve, in: J. Orchid Soc. India: 17-25.

[11] Gogoi K, RL Borah and GC Sharma, 2009, Orchid flora of Joypur Reserve Forest of Dibrugarh district of Assam, India in: Pleione, 3 (2): 135-147.

[12] Gogoi K, RL Borah and GC, Sharma, 2010, Orchid flora of Dibru-Saikhowa National Park and Biosphere Reserve, Assam, India in: Pleione 4 (1): 124-134.
[13] Hegde SN, 1984, Orchids of Arunachal Pradesh. Govt. of Arunachal Pradesh,Itanagar, India,

[14] Hooker JD, 1890, Orchidaceae. In: Flora of British India. L.Reeve and Co., Ashford, Kent. V: 687 - 864 \& VI: 1 - 198. London.

[15] Jain SK. and RR Rao, 1977, A Handbook of Field and Herbarium Methods. Today \& Tomorrow's Printers \& Publishers, New Delhi, India.

[16] King $G$ and $R$ Pantling, 1898, The Orchids of the Sikkim-Himalaya. In Annals of the Royal Botanic Garden, Calcutta.

[17] Kumar Sathish and KS Manilal, 1994. A Catalogue of Indian Orchids. Bishen Singh Mahendra Pal Singh, Dehra Dun, India.

[18] Lindley J, 1857, Contributions to the Orchidology of India-I.in: Journal of Linnean Society. 1: 170- 190.

[19] Lindley J, 1859, Contributions to the Orchidology of India-II.in: Journal of Linnean Society. 3: 1- 63.

[20] Lindley J, 1852-1855, Folia Orchidacea -An enumeration of the known species of Orchids. London.

[21] Lindley J, 1830-1840, The genera and species of Orchidaceous plants. London.

[22] Lucksom SZ, 2007, The Orchids of Sikkim and North East Himalaya: Development Area, Jiwan Thing Marg, Gangtok, East Sikkim, India.

[23] Misra S, 2007, Orchids of India. Bishen Singh Mahendra Pal Singh, Dehra Dun, India.

[24] Nath PC \& DR Das, 2011. Bulbophyllum Sikkimense (King \& Pantling) J.J. Smith (Orchidaceae) - a new record for the orchid flora of Assam, India. Pleione 5(2): 341 - 344.

[25] Pangtey YPS., SS Samant and GS Rawat, 1991, Orchids of Kumaun Himalaya. Bishen Singh Mahendra Pal Singh, Dehra Dun, India.

[26] Pearce NR and PJ Cribb, 2002, Flora of Bhutan. The Orchids of Bhutan. Vol. 3, part 3. Royal Botanic Garden, Edinburgh.

[27] Pradhan M, 2004, 100 Sikkim Himalayan Orchids, Mumbai, India,

[28] Pradhan UC, 1976, Indian Orchids Guide to Identificationand Culture. Vol- I, Bharat Lithographing Co., Calcutta, India.

[29] Pradhan UC, 1979, Indian Orchids Guide to Identification and Culture. Vol- II. Thomson Prass, Faridabad, India.

[30] Raizada MB., HB Naithani and HO Saxena, 1981, Orchids of Mussoorie. Bishen Singh Mahendra Pal Singh, Dehra Dun, India,

[31] Seidenfaden G, Notes on Cirrhopetalum Lindl.In Dansk Bot. Arkiv 29(1): 1-260, 1973.

[32] Yonzone Rajendra and A Kamran, 2008, Ethnobotanical Uses of Orchids. Abstract in an International Seminar of XVIIIth Annual Conference of IAAT October 11-13. "Multidisciplinary approaches in Angiosperm Systematics" Kalyani University, West Bengal. 